\title{
3D Shape Perception in Posterior Cortical Atrophy: A Visual Neuroscience Perspective
}

\author{
[Céline R. Gillebert, ${ }^{1,2 \star}$ Jolien Schaeverbeke, ${ }^{1 \star}$ Christine Bastin, ${ }^{3}$ Veerle Neyens, ${ }^{1}$ - Rose Bruffaerts, ${ }^{1,4}$ \\ An-Sofie De Weer, ${ }^{1}{ }^{1}$ Alexandra Seghers, ${ }^{1,4}$ Stefan Sunaert, ${ }^{5}$ Koen Van Laere, ${ }^{6}$ Jan Versijpt, ${ }^{7}$ Mathieu Vandenbulcke, ${ }^{8}$ \\ (DEric Salmon, ${ }^{3}$ James T. Todd, ${ }^{\oplus}$ Guy A. Orban, ${ }^{10,11 \dagger}$ and ${ }^{\circledR R i k}$ Vandenberghe ${ }^{1,4,12 \dagger}$ \\ ${ }^{1}$ Laboratory for Cognitive Neurology, Department of Neurosciences, KU Leuven, 3000 Leuven, Belgium, ${ }^{2}$ Department of Experimental Psychology, \\ University of Oxford, Oxford OX1 3UD, United Kingdom, ${ }^{3}$ Cyclotron Research Centre, University of Liège, 4000 Liège, Belgium, ${ }^{4}$ Neurology Department, \\ ${ }^{5}$ Radiology Department, and ${ }^{6}$ Division of Nuclear Medicine, University Hospitals Leuven, 3000 Leuven, Belgium, ${ }^{7}$ Department of Neurology, University \\ Hospital Brussels, 1090 Brussels, Belgium, ${ }^{8}$ Psychiatry Department, University Hospitals Leuven, 3000 Leuven, Belgium, ${ }^{9}$ Department of Psychology, The \\ Ohio State University, Columbus, Ohio 43210, ${ }^{10}$ Laboratory of Neuro- and Psychophysiology, Department of Neurosciences, KU Leuven, 3000 Leuven, \\ Belgium, ${ }^{11}$ Department of Neuroscience, University of Parma, 43125 Parma, Italy, and ${ }^{12}$ Alzheimer Research Centre KU Leuven, Leuven Research Institute \\ for Neuroscience and Disease, KU Leuven, 3000 Leuven, Belgium
}

Posterior cortical atrophy (PCA) is a rare focal neurodegenerative syndrome characterized by progressive visuoperceptual and visuospatial deficits, most often due to atypical Alzheimer's disease (AD). We applied insights from basic visual neuroscience to analyze 3D shape perception in humans affected by PCA. Thirteen PCA patients and 30 matched healthy controls participated, together with two patient control groups with diffuse Lewy body dementia (DLBD) and an amnestic-dominant phenotype of AD, respectively. The hierarchical study design consisted of 3D shape processing for 4 cues (shading, motion, texture, and binocular disparity) with corresponding 2D and elementary feature extraction control conditions. PCA and DLBD exhibited severe 3D shape-processing deficits and AD to a lesser degree. In PCA, deficient 3D shape-from-shading was associated with volume loss in the right posterior inferior temporal cortex. This region coincided with a region of functional activation during 3D shape-from-shading in healthy controls. In PCA patients who performed the same fMRI paradigm, response amplitude during 3D shape-from-shading was reduced in this region. Gray matter volume in this region also correlated with 3D shape-from-shading in AD. 3D shape-from-disparity in PCA was associated with volume loss slightly more anteriorly in posterior inferior temporal cortex as well as in ventral premotor cortex. The findings in right posterior inferior temporal cortex and right premotor cortex are consistent with neurophysiologically based models of the functional anatomy of 3D shape processing. However, in DLBD, 3D shape deficits rely on mechanisms distinct from inferior temporal structural integrity.

Key words: Alzheimer's disease; depth perception; fMRI; Lewy body; voxel-based morphometry

Significance Statement

Posterior cortical atrophy (PCA) is a neurodegenerative syndrome characterized by progressive visuoperceptual dysfunction and most often an atypical presentation of Alzheimer's disease (AD) affecting the ventral and dorsal visual streams rather than the medial temporal system. We applied insights from fundamental visual neuroscience to analyze 3D shape perception in PCA. 3D shape-processing deficits were affected beyond what could be accounted for by lower-order processing deficits. For shading and disparity, this was related to volume loss in regions previously implicated in 3D shape processing in the intact human and nonhuman primate brain. Typical amnestic-dominant AD patients also exhibited 3D shape deficits. Advanced visual neuroscience provides insight into the pathogenesis of PCA that also bears relevance for vision in typical AD. 


\section{Introduction}

Visual neuroscience has made big strides over the past decades. The translation of these basic advances into a better understanding of neurological phenotypes remains an important area of research. One of the most complex visual disorders in neurology is posterior cortical atrophy (PCA) (Benson et al., 1988; Berthier et al., 1991; Graff-Radford et al., 1993; Caine, 2004; Tang-Wai et al., 2004; Lehmann et al., 2011a, 2011b; Crutch et al., 2012), a rare syndrome that is characterized by progressive visuoperceptual disturbances and is most often due to Alzheimer's disease (AD). Markers of cerebral amyloidosis are most often indistinguishable from what is seen in typical AD (Rosenbloom et al., 2011; de Souza et al., 2011; Lehmann et al., 2013a). The predominance of visual complaints and symptoms is attributable to the unusual distribution of neurofibrillary tangles (NFTs) and neuronal loss in primary and secondary visual areas (Hof et al., 1990; Hof and Bouras, 1991; Levine et al., 1993; Hof et al., 1997; Tang-Wai et al., 2004; von Gunten et al., 2006; Kas et al., 2011; Rosenbloom et al., 2011; Lehmann et al., 2013a, 2013b; Ossenkoppele et al., 2015). The topography of the neuropathological damage is heterogeneous, with variable involvement of dorsal or ventral areas of the visual processing stream (Levine et al., 1993; Hof et al., 1997; Migliaccio et al., 2012). Although deficits in 3D perception have been mentioned in the context of PCA (Rogelet et al., 1996), to the best of our knowledge, 3D shapeprocessing deficits in PCA have not been analyzed in a systematic and neuroscientifically grounded manner.

The way in which the brain computes $3 \mathrm{D}$ shape from a $2 \mathrm{D}$ retinal image has been largely elucidated based on single-cell recordings in nonhuman primates combined with functional imaging of the intact human and nonhuman primate brain (for review, see Orban, 2011). The 3D shape-processing system involves both the dorsal and ventral processing stream. The relative contribution of each of these streams depends on the cue defining 3D shape: 3D shape-from-shading relies more heavily on the inferior temporal cortex and 3D shape-from-motion mainly on more dorsal processing areas (Georgieva et al., 2008; Orban, 2011), whereas 3D from texture and disparity activate both dorsal and ventral processing stream areas (Orban, 2011).

Within the visual processing hierarchy, the input to the 3D shape-processing system relies on the output of areas extracting elementary features. These features are also used to extract contours. For the luminance cue, the orientation of these contours is

Received Sept. 2, 2014; revised June 20, 2015; accepted July 22, 2015.

Author contributions: C.R.G., G.A.O., and R.V. designed research; C.R.G., J.S., C.B., V.N., R.B., A.S.D.W., A.S., S.S., K.V.L., J.V., M.V., E.S., J.T.T., G.A.O., and R.V. performed research; R.V. contributed unpublished reagents/analytic tools; C.R.G., J.S., R.B., J.T.T., G.A.O., and R.V. analyzed data; C.R.G., J.S., C.B., J.T.T., G.A.O., and R.V. wrote the paper.

This work was supported by Inter-University Attraction Pole P6/29 (R.V., E.S., G.A.O.) and P7/11 (R.V., E.S., G.A.O.), KU Leuven (Grant 0T/12/097 and Programme Financing to R.V.), Fund for Scientific Research-Flanders (FW0; Grant G.0660.09N to R.V.), Fund for Scientific Research of the Wallonia-Brussels Federation (E.S.) and Stichting Alzheimer Onderzoek (Grant 11020 to R.V.), Vlaams Initiatief voor Netwerken voor Dementie Onderzoek (R.V.), and Transformationeel Geneeskundig Onderzoek BioAdaptAD (R.V.). R.V. and K.V.L. are senior clinical investigators of the FWO. C.R.G. holds a Sir Henry Wellcome Postdoctoral Fellowship funded by the Wellcome Trust (Grant 098771/Z/12/Z). We thank the patients and their families for their participation and Wouter Depuydt for technical support.

The authors declare no competing financial interests.

${ }^{*}$ C.R.G. and J.S. are co-first authors.

†G.A.O. and R.V. are co-senior authors.

This article is freely available online through the J Neurosci Author Open Choice option.

Correspondence should be addressed to Dr. Rik Vandenberghe, Neurology Department, University Hospitals Leuven, Herestraat 49, Box 7003, 3000 Leuven, Belgium. E-mail: rik.vandenberghe@uz.kuleuven.ac.be.

DOI:10.1523/JNEUROSCI.3651-14.2015

Copyright @ 2015 Gillebert, Schaeverbeke et al.

This is an Open Access article distributed under the terms of the Creative Commons Attribution License Creative Commons Attribution 4.0 International, which permits unrestricted use, distribution and reproduction in any medium provided that the original work is properly attributed. extracted at the level of V1 (De Valois et al., 1982) and, for nonluminance cues, at higher levels of the ventral stream, as shown in nonhuman primate (Sáry et al., 1995; Mysore et al., 2006; Mysore et al., 2010; El-Shamayleh and Movshon, 2011) and human (DuPont et al., 1997; Mendola et al., 1999; Tyler et al., 2006) studies. We therefore applied a hierarchical experimental design with three levels: (1) 3D shape processing from shading, texture, motion, and binocular disparity; (2) 2D shape processing from the same cues; and (3) processing of the elementary features.

We included two patient control groups for two distinct reasons. The clinical phenotype of diffuse Lewy body dementia (DLBD) is known to be associated with visuoperceptual deficits (Mori et al., 2000; Calderon et al., 2001; Mosimann et al., 2004). This allowed us to assess how disease specific the visuoperceptual deficits observed in PCA and their neuroanatomical underpinnings were. As our second patient control group, we included a group of patients with typical AD (Dubois et al., 2014). By "typical AD," we refer to the most common form of AD that initially presents with an amnestic syndrome (McKhann et al., 2011; Dubois et al., 2014). Including a typical AD group allowed us to ascertain the relevance of the findings obtained in the PCA for the far more common amnestic $\mathrm{AD}$ phenotype.

We used 3D stimuli and behavioral tasks similar to those used in the previous studies (Todd, 2004; Georgieva et al., 2008; Nelissen et al., 2009) and related the deficits to regional measures of cerebral volume loss. The anatomical correlations may provide a better understanding of the pathogenetic mechanisms leading to visual dysfunction in PCA and shed light on the contribution of cortical regions to $3 \mathrm{D}$ shape processing in the intact brain.

\section{Material and Methods}

\section{Subjects}

The study was approved by the Ethics Committee of University Hospitals Leuven. All participants provided written informed consent in accordance with the Declaration of Helsinki. All participants had normal or corrected-to-normal vision except for PCA Case 6, who had a congenital left monocular blindness.

A consecutive academic memory clinic-based series of 16 patients who received a diagnosis of PCA due to $\mathrm{AD}$ were screened for participation in this research study. Based on performance of prior test runs (see below), three patients were excluded, leaving 13 patients who were eligible to undergo the full protocol (two men, 11 women). The study started as a monocentric study run at the academic memory clinic of University Hospitals Leuven ( $n=8$, Cases $1-7$ and 13). In a second phase, additional subjects were recruited via the Liège University Hospital ( $n=4$, Cases $8-11)$ and the University Hospital Brussels $(n=1$, Case 12) (Table 1). The clinical diagnosis was based on the standard procedures as implemented at the different academic centers. In all cases, the presenting symptom consisted of visuoperceptual complaints with insidious onset and slow progression and preserved insight. The impact on instrumental activities of daily living was caused by the visuoperceptual disturbances (such as reduced speed of reading, difficulties with car driving under perceptually degraded conditions such as rainy weather, falls due to misperception of uneven surfaces such as stairs, etc.). Apart from a clinical evaluation by an academic neurologist (R.V., E.S., or J.V.), investigations consisted of a clinical structural MRI, a conventional neuropsychological assessment and, in 11 of 13 cases, a clinical $\left[{ }^{18} \mathrm{~F}\right]$-fluorodeoxyglucose positron emission tomography $\left(\left[{ }^{18} \mathrm{~F}\right]-\mathrm{FDG}-\mathrm{PET}\right) \mathrm{scan}$. The clinical diagnosis of PCA was confirmed by the distribution of FDG-PET hypometabolism. In all patients who received an $\left[{ }^{18} \mathrm{~F}\right]$-FDG-PET, hypometabolism was located predominantly in posterior cortex. The laterality of the lesions varied between cases, as well as their extent. In Cases 6-9 and 13, the premotor cortex was also involved. The individuals' MRI volume loss was consistent with the FDG-PET hypometabolism confirming the mainly posterior involvement, but with a 
Table 1. PCA patients: demographics and clinical-technical investigations

\begin{tabular}{|c|c|c|c|c|c|c|c|c|c|c|c|c|c|}
\hline & Case 1 & Case 2 & Case 3 & Case 4 & Case 5 & Case 6 & Case 7 & Case 8 & Case 9 & Case 10 & Case 11 & Case 12 & Case 13 \\
\hline Age & 68 & 67 & 56 & 63 & 52 & 51 & 57 & 65 & 54 & 61 & 71 & 53 & 66 \\
\hline Sex & $\mathrm{F}$ & M & M & $\mathrm{F}$ & $\mathrm{F}$ & $\mathrm{F}$ & $\mathrm{F}$ & $\mathrm{F}$ & $\mathrm{F}$ & M & $\mathrm{F}$ & $\mathrm{F}$ & $\mathrm{F}$ \\
\hline Disease duration (y) & 1.5 & 2 & 1 & 1.5 & 1.5 & 2 & 1 & 0.75 & 0.5 & 0.5 & 2 & 3.75 & 3 \\
\hline MMSE & 27 & 28 & 22 & 26 & 19 & 24 & 19 & 17 & 20 & 21 & 26 & 22 & 23 \\
\hline \multicolumn{14}{|l|}{ Measures of topography } \\
\hline Structural MRI & + & + & + & + & + & + & + & + & + & + & + & + & + \\
\hline FDG-PET & + & + & + & + & - & + & + & + & + & + & + & - & + \\
\hline \multicolumn{14}{|l|}{ Amyloid biomarkers } \\
\hline PIB PET SUVR comp (cutoff = 1.43) & 2.17 & - & - & - & 1.92 & - & 2.20 & - & - & - & - & - & - \\
\hline $\operatorname{CSF} A \beta_{42}(\mathrm{pg} / \mathrm{ml})($ cutoff $=600 \mathrm{pg} / \mathrm{ml})$ & 312 & 337 & 389 & - & 581 & 239 & - & - & - & - & - & 359 & 457 \\
\hline
\end{tabular}

SUVR $_{\text {comp }}$, Standardized uptake value ratio in the composite cortical volume of interest; - , test not performed.

lower sensitivity. In the two patients in whom no FDG-PET was available (Cases 5 and 12), posterior gray matter loss was present on the MRI scan. CSF AD biomarkers (ELISA; Fujirebio Europe) and Pittsburgh Compound B ([ $\left.\left.{ }^{11} \mathrm{C}\right]-\mathrm{PIB}\right)$ amyloid PET were performed in 7 and 3 patients, respectively, and confirmed AD as etiology (Table 1). In all patients who received a lumbar puncture, amyloid-beta 42 $\left(\mathrm{A} \beta_{42}\right)$ was $<500 \mathrm{pg} / \mathrm{ml}$, except Case 5 , in whom $\mathrm{A} \beta_{42}=581 \mathrm{pg} / \mathrm{ml}$ with a pathological tau level of $498 \mathrm{pg} / \mathrm{ml}$, which was therefore clearly also within the AD range. Clinical follow-up currently extends 3-6 years after the study and the clinical evolution was fully compatible with the diagnosis of PCA in all cases, with the development of severe disability due to virtual blindness in a more advanced stage. None of these subjects have developed a corticobasal or other extrapyramidal neurodegenerative syndrome over time.

Thirty cognitively intact age-matched controls ( 17 men, 13 women, age $61 \pm 5$ years) participated in the psychophysical experiment. Controls were recruited through advertisement in a regional newspaper asking for volunteers above the age of 55 years for participation in a scientific study. Control participants did not have any subjective memory or visual complaints or any history of significant neurological or psychiatric illness. None of the control participants took any psychotropic or vasoactive medication.

Twenty-four of these controls and 94 additional cognitively intact age-matched controls ( 50 at the KU Leuven site, 44 at the Liège site) also received a volumetric MRI ( 60 men, 58 women, age $65 \pm 7.32$ years, range 52-85).

As a patient control group, we included a consecutive series of 13 patients who fulfilled the consensus criteria for clinically probable dementia with Lewy bodies (McKeith et al., 2005) [11 men, 2 women, age $69 \pm 6.1$ years, disease duration $2.4 \pm 1.5$ years, Mini-Mental State Examination (MMSE) score $24.5 \pm 3.1$ ]

As a further patient control group, we included a consecutive series of 14 patients with amnestic MCI due to AD or early-stage clinically probable AD ( 11 men, 3 women, $66 \pm 6.8$ years, MMSE score $25.1 \pm$ 3.7) who were recruited through the memory clinic of University Hospitals Leuven.

According to one-way ANOVA, the 3 patient groups did not differ in MMSE score $\left(F_{(2,39)}=1.95, p=0.16\right)$. Age differed significantly between the 4 groups $\left(F_{(3,66)}=8.04, p<0.001\right)$ : PCA patients did not differ in age from the healthy controls $(p=0.70)$, but were younger than the typical AD patients $(p=0.01)$ and DLBD patients $(p<0.001)$.

\section{Psychophysical experiments}

All 13 PCA patients, the 13 DLBD and 14 AD patients, as well as 30 healthy controls, participated in the psychophysical experiments. All controls underwent the full hierarchical design except for four of them, who only received the $3 \mathrm{D}$ tests.

Stimulus presentation and response registration were controlled by a PC running Presentation 11.3 (Neurobehavioral Systems) and inhouse software written in C. Participants were seated $50 \mathrm{~cm}$ from a cathode ray tube monitor (diagonal measurement $48.26 \mathrm{~cm}$, resolution $1024 \times 768$ pixels, refresh rate $75 \mathrm{~Hz}$ ).

The psychophysical experiments were organized according to a hierarchical design with three levels. At the 3D level, we evaluated the pro- cessing of 3D shape-from-shading, texture, motion, or binocular disparity cue (Fig. $1 A-C$ ). At the $2 \mathrm{D}$ level, we evaluated orientation discrimination of rectangular gratings defined by luminance, texture, motion, or disparity cues (Fig. 1D). At a third, basic level, we evaluated elementary feature processing of the four cues (Fig. $1 E$ ).

$3 D$ shape perception. The visual stimuli were created and rendered in four modalities (3D shape-from-shading, shape-from-texture, shapefrom-motion, and shape-from-disparity) using 3D Studio Max by Autodesk. The 3D shape-from-shading and the 3D shape-from-texture stimuli (Fig. $1 A-C$ ) were created as described in Georgieva et al. (2008). The 3D shape-from-motion and 3D shape-from-disparity stimuli (Fig. $1 A$ ) were filled with an isotropic and statistically homogeneous texture pattern. The 3D shape-from-motion stimuli oscillated back and forth around a vertical axis at a rate of $87.5 \%$ (Norman et al., 2004). The 3D shape-from-disparity stimuli were displayed binocularly through red/ blue anaglyph glasses.

The stimuli depicted 10 randomly generated complex 3D surfaces, representing the front surface of meaningless $3 \mathrm{D}$ objects, with a large assortment of variably shaped hills, ridges, valleys, and dimples at multiple scales (Fig. 1B,C) (Norman and Todd, 1995; Fleming et al., 2004; Norman et al., 2004; Todd, 2004; Georgieva et al., 2008). Because the surfaces were smoothly curved, they did not provide information from configurations of edges and vertices (Moore and Engel, 2001; Kourtzi et al., 2003). Depth maps corresponding to three representative surfaces indicate that, relative to the global maximum of the 3D surface (point nearest to the subject), the border was located $\sim 5 \mathrm{~cm}$ further back in depth (Fig. 1B). Compared with the global maximum of each 3D surface, the depth values present in the surfaces averaged $1.89 \mathrm{~cm}$ (SD across all points of all 3D surfaces, $1.03 \mathrm{~cm}$ ), with the extremes of the distribution corresponding to the border of the surfaces (Fig. 1C).

Before initiation of the experimental runs, four test trials were provided per cue. In the first two trials, the experimenter pointed the five fingers of her left or right hand to the subject and the subject had to indicate which fingers were closest to him/her (i.e., the middle finger). These test trials did not require accurate depth perception because they could be solved based on prior knowledge and were meant to clarify the concept of proximity. In the last two test trials, subjects performed the 3D shape task on a 3D surface on the computer screen. The 3D surfaces used for the test trials differed from those used in the subsequent experimental runs. Subjects who failed the first two test trials, who were not sure about the location of the local maxima in the last two trials, or were unable to verbally repeat the task instructions for the last two test trials were excluded from the study.

In the experimental runs, the four cues (shading, texture, motion, and disparity) were presented in a blocked design, one block per cue. The 10 $3 \mathrm{D}$ surfaces were presented in random order in a block. The order of the blocks was counterbalanced across participants. When 3D structure was induced by a monocular cue (shading, texture, or motion), participants wore a patch over the nondominant eye. When the $3 \mathrm{D}$ structure was induced by binocular disparity, the participants wore red/blue anaglyph glasses. Each trial was initiated by pressing "RETURN" on the keyboard. An image of a 3D shape (average size, $12^{\circ}$ ) appeared at the center of the screen on a blue (motion, shading, texture) or black (disparity) back- 


\section{A Extraction of 3D shape}

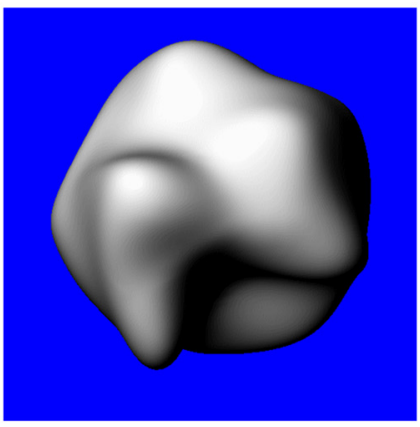

shading

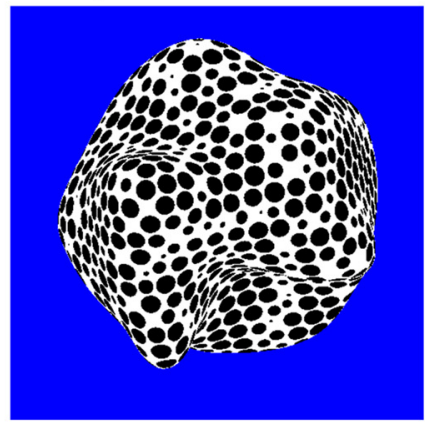

texture

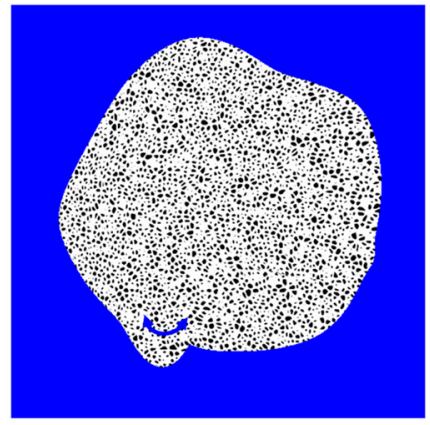

motion

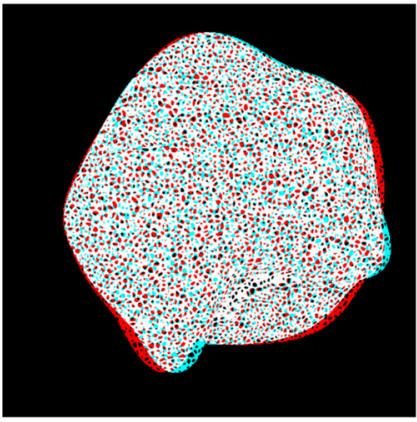

disparity

\section{B Illustration of depth maps}
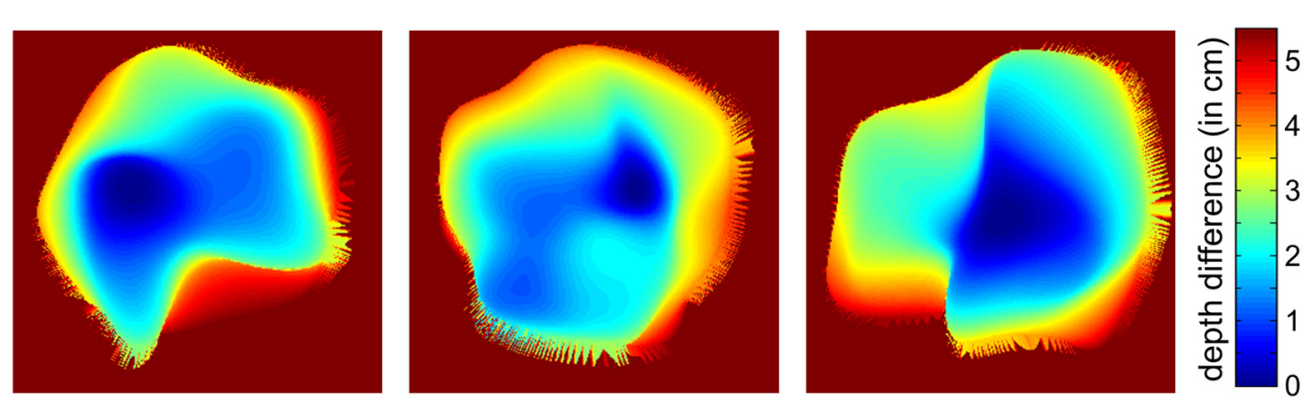

\section{c Distribution of depth differences}

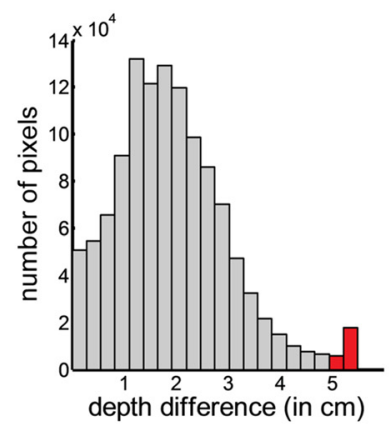

\section{Extraction of 2D shape}
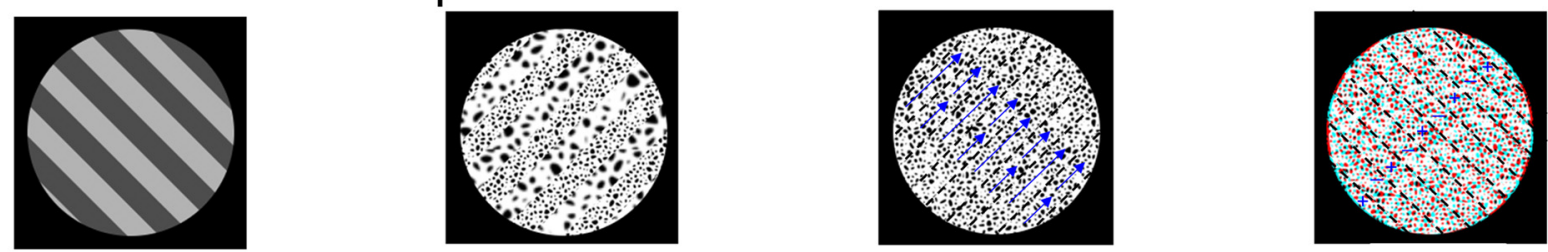

\section{E Extraction of features}

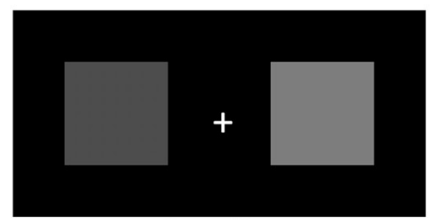

shading

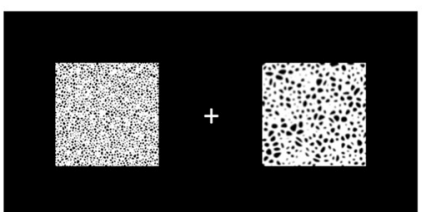

texture

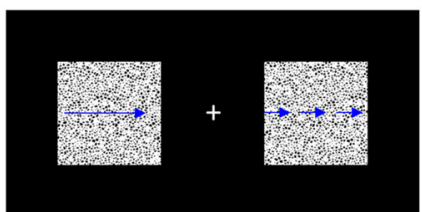

motion

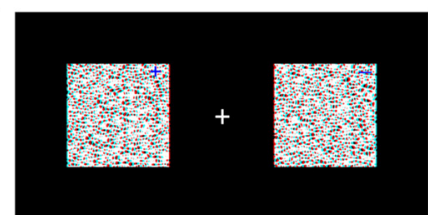

disparity

Figure 1. Visual stimuli. $\boldsymbol{A}-\boldsymbol{C}$, Visual stimuli of the psychophysical experiments. $\boldsymbol{A}$, Extraction of $3 \mathrm{D}$ shape-from-shading, texture, motion, and binocular disparity. $\boldsymbol{B}$, Examples of the depth maps for three 3D surfaces used in the psychophysical experiment. The color bar reflects the depth difference in centimeters compared with the global maximum of the surface (cutoff at $5.5 \mathrm{~cm}$ because depth at the very border is unstable). C, Histogram of the depth differences across all pixels of all $103 \mathrm{D}$ surfaces. The extreme values (red) correspond to the borders of the surfaces (those exceeding $5.5 \mathrm{~cm}$ have been set to $5.5 \mathrm{~cm}$ ). D. Extraction of $2 D$ shape with the neighboring bars of the grating differing in luminance, texture, motion, or disparity. $E$, Extraction of features with two squares differing in luminance, texture, motion, or disparity.

ground until the next trial was initiated. Participants were allowed to make eye movements and were instructed to indicate the global maximum of the 3D structure (point nearest to them) as accurately as possible by displacing a red cross using the mouse. If the participant was not able to manipulate the mouse, he/she could indicate the global maximum with a pen and the experimenter would move the red cross to that location until the participant had approved it verbally.

As our primary outcome analysis, we calculated the error-in-depth (in centimeters) as follows. The difference between the global maximum indicated by the subject and the true global maximum can be mathematically described as a 3D vector that represents the error in the $3 \mathrm{D}$ shape perception (the 3D shape error vector). Error-in-depth is the projection of this vector onto the sagittal plane. We then conducted a two-way mixed ANOVA with group (four levels: healthy control participants, PCA, DLBD, and AD) as the between-subjects factor and cue (four levels: shading, texture, motion, and disparity) as the within-subjects factor (threshold: $p<0.05$ ). When sphericity could not be assumed (Mauchly's sphericity test: $p<0.05$ ), $p$ values were adjusted using the Greenhouse-Geisser correction. Post hoc comparisons consisted of the comparison between PCA and each of 
3D shape from shading

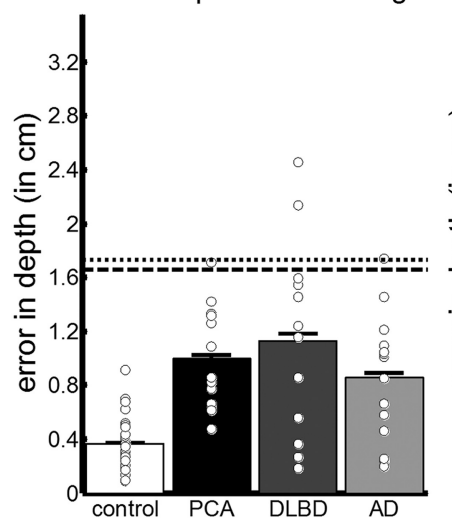

3D shape from texture

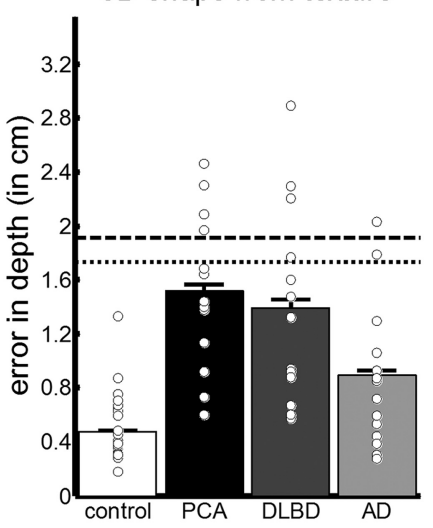

3D shape from motion

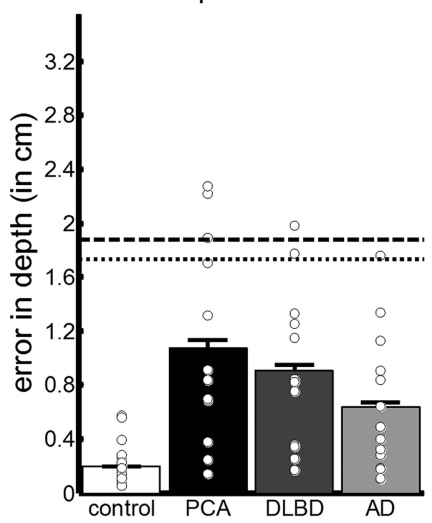

3D shape from disparity

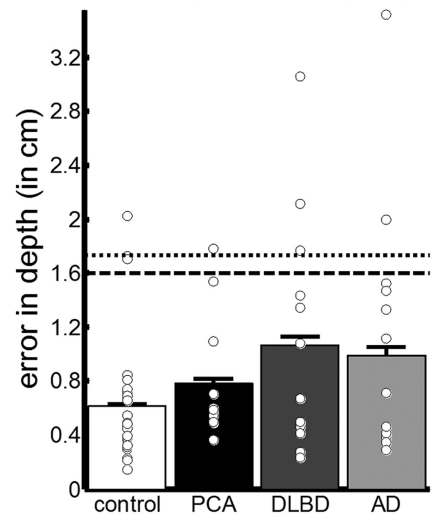

...... mean error-in-depth based on 1000 random responses per 3D surface

- - mean error-in-depth when visuomotor deficits are simulated in control participants

Figure 2. Primary outcome analysis based on error-in-depth. Behavioral performance for healthy control participants (white), PCA patients (black), DLBD patients (dark gray), and typical AD patients (light gray) for shading, texture, motion, and binocular disparity. Behavioral performance is expressed as the depth component of the difference between the global maximum indicated by the observer and the true global maximum (error-in-depth). Smaller values on this measure reflect better performance. The dotted line indicates the average value on this measure based on 1000 responses randomly distributed across the interior zone of each 3D surface. The dashed line reflects the average error-in-depth obtained when a random spatial error is added to the control data to simulate motor deficits that may be present in PCA patients. Error bars indicate 1 SEM across participants. The circles indicate the individual data points.

Table 2. Psychophysical experiments: behavioral data

\begin{tabular}{|c|c|c|c|c|c|c|c|c|c|c|c|c|c|}
\hline \multirow[b]{2}{*}{ Group } & \multirow[b]{2}{*}{ Case } & \multicolumn{4}{|c|}{ Extraction of 3D shape } & \multicolumn{4}{|c|}{ Extraction of 2D shape } & \multicolumn{4}{|c|}{ Extraction of features } \\
\hline & & Shading & Texture & Motion & Disparity & Luminance & Texture & Motion & Disparity $^{b}$ & Luminance & Texture & Motion & Disparity $^{b}$ \\
\hline \multirow[t]{11}{*}{ PCA } & 1 & 0.48 & 1.38 & 1.31 & 0.60 & -2.19 & -0.63 & -0.56 & -1.68 & -0.24 & -1.05 & -0.11 & 0.80 \\
\hline & 3 & 1.42 & 1.64 & 0.68 & 0.70 & -0.88 & 0.66 & 0.61 & & -0.14 & -0.60 & 0.57 & \\
\hline & 4 & 0.64 & 0.60 & 0.38 & 0.56 & -1.41 & -0.18 & 0.40 & & -0.93 & -0.86 & -0.56 & 0.05 \\
\hline & 5 & 1.33 & 1.97 & 0.91 & 0.54 & -0.06 & 0.79 & 0.36 & 0.33 & -0.02 & -0.37 & -0.12 & -0.31 \\
\hline & $\underline{6}^{a}$ & 0.78 & 1.44 & 0.25 & & -1.14 & -0.03 & 0.49 & & -0.35 & -1.07 & -0.40 & \\
\hline & 9 & 1.71 & 2.46 & 2.22 & 1.54 & -0.40 & 0.62 & 0.42 & 0.45 & 0.52 & -0.41 & 0.04 & \\
\hline & 10 & 0.82 & 2.30 & 2.28 & 1.10 & -1.15 & 0.01 & -0.06 & -1.16 & 0.60 & 0.42 & 0.61 & 0.08 \\
\hline & 11 & 0.86 & 1.14 & 0.70 & 0.49 & -1.31 & 0.71 & -0.55 & 0.51 & -0.61 & -1.02 & -0.42 & -0.93 \\
\hline & $\underline{12}$ & 0.66 & 2.09 & 1.89 & 0.50 & -0.84 & -0.43 & 0.53 & -0.61 & -0.34 & -0.54 & -0.24 & -0.95 \\
\hline & $\overline{13}$ & 0.62 & 0.73 & 0.14 & 0.37 & -1.58 & -0.75 & 0.52 & & 0.25 & -0.41 & -0.27 & \\
\hline & Mean & 1.00 & 1.52 & 1.08 & 0.78 & -1.12 & 0.00 & 0.29 & -0.31 & -0.07 & -0.62 & -0.15 & -0.32 \\
\hline DLBD & Impaired $(n)$ & 9 & 9 & 9 & 4 & 6 & 4 & 4 & 0 & 2 & 3 & 5 & 1 \\
\hline \multirow[t]{3}{*}{$A D$} & Mean & 0.85 & 0.86 & 0.62 & 1.03 & -1.50 & -0.30 & -0.56 & -1.30 & -0.72 & -0.77 & -0.41 & -0.57 \\
\hline & SEM & 0.13 & 0.13 & 0.14 & 0.24 & 0.12 & 0.13 & 0.25 & 0.10 & 0.06 & 0.10 & 0.09 & 0.17 \\
\hline & Impaired $(n)$ & 8 & 5 & 7 & 4 & 7 & 4 & 4 & 0 & 3 & 4 & 7 & 0 \\
\hline \multirow[t]{2}{*}{ Controls } & Mean & 0.37 & 0.48 & 0.19 & 0.61 & -1.88 & -0.77 & -1.17 & -0.49 & -0.83 & -1.20 & -0.79 & -0.29 \\
\hline & SEM & 0.04 & 0.04 & 0.02 & 0.08 & 0.04 & 0.06 & 0.11 & 0.20 & 0.04 & 0.04 & 0.04 & 0.12 \\
\hline Significant group difference & & Yes & Yes & Yes & No & Yes & Yes & Yes & No & Yes & Yes & Yes & No \\
\hline
\end{tabular}

Extraction of $3 \mathrm{D}$ shape. Test scores reflect the error-in-depth (in centimeters) between the global maximum indicated by the participant and the true global maximum; extraction of $2 \mathrm{D}$ shape and extraction of features, test scores reflect the contrast between neighboring bars of the gratings (expressed as logarithmic Michaelson contrasts) to achieve an average performance of $82 \%$. Test scores in bold indicate significantly decreased performance compared with the control population (one-sided, $p<0.05$ ) (Crawford and Garthwaite, 2002). Cases 1, 2, 6, 7, and 12 (underlined) participated in the fMRl experiment.

${ }^{a}$ Only monocular cues were tested in Case 6, who was congenitally blind in one eye.

${ }^{b}$ For the disparity-defined stimuli, we observed an interaction between the time needed to perceive stereograms and the QUEST procedure. This resulted in the inability to define a threshold in some participants (indicated by an empty cell) and a less reliable threshold in the other participants.

the control groups for each of the cues corrected for the number of cues and contrasts $(n=12)$.

A complementary and well established way of analyzing the responses per group for this type of 3D shape-processing task relies on the in-plane distribution of the responses (Todd et al., 2001; Koenderink et al., 2002). This amounts to an analysis of the frontoparallel components of the
3D shape error vector. We calculated covariance ellipses encompassing $95 \%$ of the responses per group for each 3D surface (see Fig. $3 A$ for an illustration of these ellipses) and averaged these ellipses over the 10 $3 \mathrm{D}$ surfaces. We analyzed the location of the ellipse center ( $x$ - and $y$-coordinates), the size of the ellipse (mean of the two eigenvalues), and its elongation (largest eigenvalue divided by smallest eigenvalue) (Fig. 
A

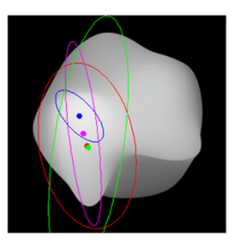

- healthy controls

- PCA patients

DLBD patients

AD patients
B

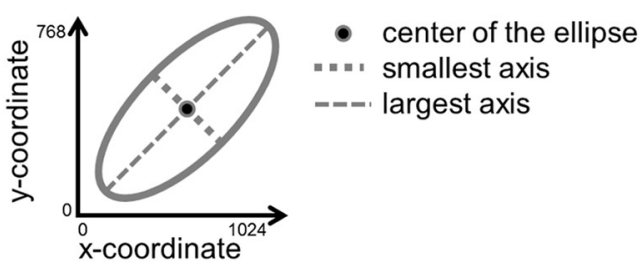

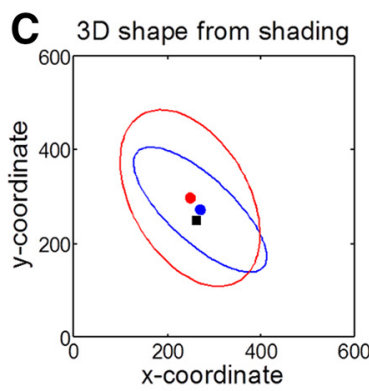

D 3D shape from shading

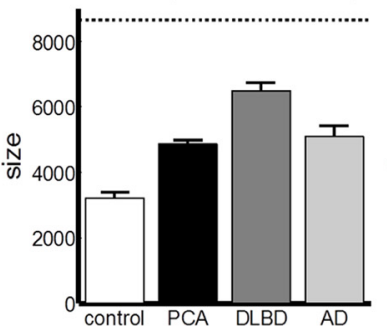

E 3D shape from shading

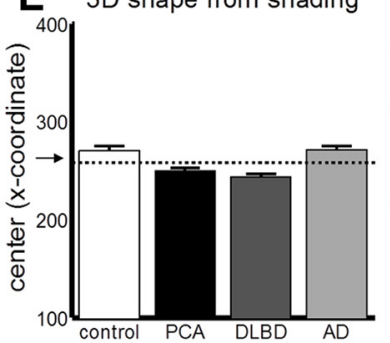

F 3D shape from shading

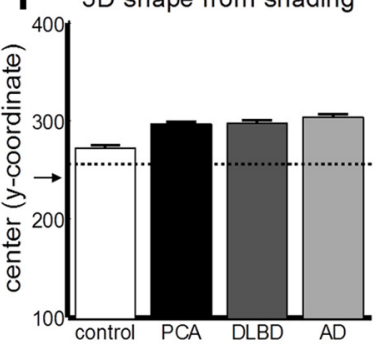

G 3D shape from shading

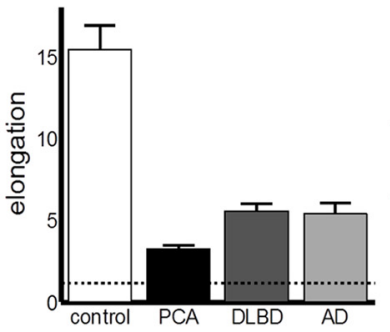

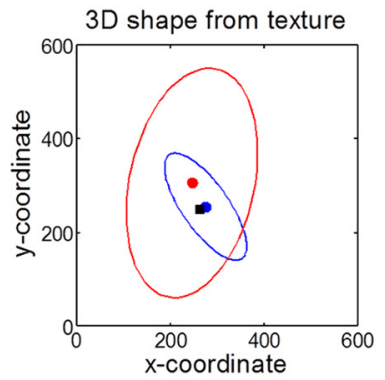
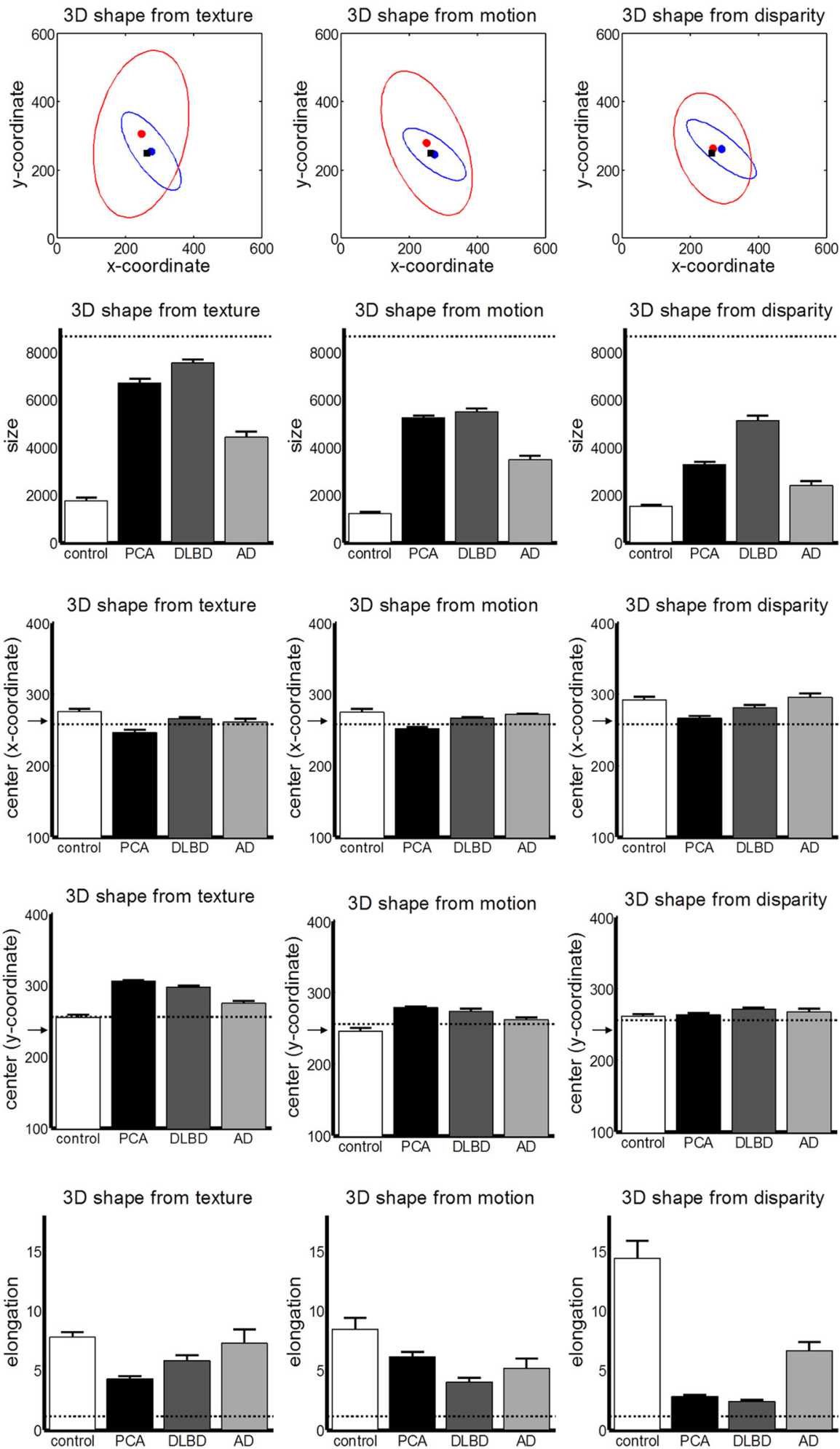

Figure 3. Analysis based of the in-plane distribution of responses. $A$, Example of a $95 \%$ covariance ellipse for healthy controls (blue), PCA patients (red), DLBD patients (green), and AD patients (magenta) superimposed on the depth map of a 3D shape-from-shading surface. B, Illustration of the ellipse parameters. C, Average ellipses for PCA patients (red) (Figure legend continues.) 
$3 B)$. The $x$ - and $y$-coordinates correspond to the group average of the global maxima indicated by the members of the group projected in the frontoparallel plane (as opposed to the sagittal plane) and has to be referenced to the projection of the true global maximum in the frontoparallel plane. Size is a measure of response variability within a group; elongation is a measure of stimulus-induced bias in the distribution of the responses, for example, that induced by ridges. These parameters were defined for the single-3D-surface ellipses. Each of the parameters was analyzed using a two-way repeated-measures ANOVA across 3D surfaces with group (four levels) and cue (four levels) as factors at a significance threshold of $p<0.05$, corrected for the number of ellipse parameters analyzed; therefore, the uncorrected $p<0.01$. Post hoc comparisons consisted of the comparison between PCA and each of the control groups. The significance level for the post hoc comparisons was corrected for the number of comparisons per parameter $(n=12)$.

The above analyses allowed us to determine differences between PCA and the control groups, but it is also pertinent to ascertain whether performance in PCA differed from random. We randomly selected 1000 locations on the 3D surface within an interior zone, avoiding the edges of the surface, and calculated the distribution of the distances in depth. We compared the random error-in-depth to the error-in-depth obtained from the PCA patients for each cue using a Mann-Whitney $U$ test (threshold: uncorrected $p<0.05$ ). We also derived from the random distributions a covariance ellipse and compared the parameters of this covariance ellipse with that obtained in PCA.

Furthermore, we evaluated whether performance in PCA differed from what one would expect if the deviation of responses in PCA compared with controls were due to a random motor error rather than a perceptual error. We randomly generated 30 data points on a 3D surface (equaling the sample size of the group of healthy controls) and adjusted their $x$ - and $y$-coordinates such that the center of the corresponding covariance ellipse equaled that of the healthy controls (simulating normal detection of the global maximum), but its elongation and size matched those of PCA patients (simulating the variance observed in PCA under the assumption that this variance would be fully due to hypothetical motor problems). We used a Mann-Whitney $U$ test (threshold: uncorrected $p<0.05$ ) to compare the error-in-depth (relative to the global maxima) of the 30 data points and that obtained in the PCA patients. This procedure was repeated 1000 times for each cue. We calculated in which percentage of iterations the responses in PCA differed from the simulated data points.

$2 D$ orientation discrimination and elementary feature extraction. In an orientation discrimination task, observers had to decide whether a rectangular grating shown in a circular aperture (diameter $6^{\circ}$, spatial frequency $0.8^{\circ}$, random phase) at the center of the screen was tilted $45^{\circ}$ clockwise or anticlockwise from the vertical meridian in a twoalternative forced-choice procedure. The orientation of the grating was defined by discontinuities in luminance, texture coarseness, speed of motion (Sáry et al., 1994), or disparity (Fig. 1D).

To test elementary feature extraction, subjects performed a 2 -alternative forced choice task. Two squares (size $6^{\circ} \times 6^{\circ}$ ) differing in one feature (luminance, coarseness of texture, speed of motion, or disparity) were presented simultaneously $6.8^{\circ}$ to the left and to the right on the horizontal meridian (Fig. 1E). Participants were instructed to indicate the brightest shape, the

$\leftarrow$

(Figure legend continued.) and healthy controls (blue) for the four cues, with red and blue squares indicating average ellipse centers and black square average true global maxima. $\mathbf{D}-\mathbf{G}$, Parameters of the covariance ellipses (averaged over the 103D surfaces) encompassing $95 \%$ of the global maxima indicated by healthy controls (white), PCA patients (black), DLBD patients (dark gray), and AD patients (light gray) for shading, texture, motion, and binocular disparity. $C$, Size (average of the largest and the smallest eigenvalue), $\boldsymbol{D}, x$-coordinate of the ellipse center (in pixel coordinates, a pixel corresponding to $2.55 \mathrm{~min}$ of arc). The arrow indicates the coordinate of the true global maximum. $\boldsymbol{E}, y$-coordinate of the center (in pixel coordinates). The arrow indicates the coordinate of the true global maximum. $\boldsymbol{F}$, Elongation (largest eigenvalue divided by smallest eigenvalue). The dotted line indicates the average value on this measure based on 1000 responses randomly distributed across the interior zone of each 3D surface. Error bars indicate 1 SEM across participants. shape filled with the coarsest texture, the shape with the fastest motion, or the shape closest in depth.

Before initiation of the experimental runs, three test trials with maximum contrast and unlimited exposure were provided for the $2 \mathrm{D}$ shape-processing and feature extraction task for each of the four cues. Subjects who failed the test trials were excluded. In the experimental runs, the four cues (luminance, texture, motion, and disparity) were presented in a blocked design. In a trial, stimuli were presented for $800 \mathrm{~ms}$ after an auditory warning cue $(50 \mathrm{~ms})$ and a brief interval ( 400 $\mathrm{ms})$. For the tasks with the disparity cue, the stimuli were repeatedly presented for $800 \mathrm{~ms}$ until the participant made a response. Participants held a response box in their preferred hand and were instructed to press one of two keys depending on the target orientation or the position of the target square and depending on the task. In patients who, according to the test trials, were unable to press a key (left or right), the following options were evaluated for response modality: to say left or right aloud, to lift their left or right hand, or to point to one or the other stimulus shown on a piece of paper in front of them. A new trial was initiated after a response or after $4 \mathrm{~s}$ if no response was made. A block ended after 60 trials with responses.

For each cue, we determined the logarithm of the Michaelson contrast (Michaelson, 1927) between luminance levels, texture coarseness, speed of motion, and disparity needed for $82 \%$ accuracy using a single QUEST procedure (Watson and Pelli, 1983). The thresholds, expressed as log of these contrasts, were submitted to an ANOVA with group (four levels: healthy control participants, PCA patients, DLBD patients, and typical $\mathrm{AD}$ patients) as the between-subjects factor and cue as the withinsubjects factor.

Behavioral analysis at the individual level. Apart from the betweengroup comparisons, we also determined which of the individual PCA patients had a pathological error-in-depth score for the 3D tasks compared with healthy controls using a one-tailed modified $t$ test (Crawford and Garthwaite, 2002), as well as how many of the individual PCA patients showed a dissociation between $3 \mathrm{D}$ tests, a dissociation meaning that the difference in performance between two tasks in an individual patient was larger than that expected based on the difference in healthy controls $(p<0.05)$ (Crawford and Garthwaite, 2005).

In addition, we calculated diagnostic power for each $3 \mathrm{D}$ test by means of area under the curve (AUC) values obtained with receiver operator curves to discriminate between PCA and controls and between PCA and each of the patient control groups (DLBD and typical AD patients).

Relationship between $3 D$ and lower-order deficits. To evaluate to which degree lower-order deficits contributed to the 3D shape deficit in PCA patients, we performed for each cue a hierarchical multiple linear regression with error-in-depth from the global maximum as the dependent variable and the contrast thresholds on the lower-order tasks as the independent variables. Probability of $F$ to enter the model was set at $<0.05$ with probability to remove set to $>0.10$.

For those cues for which lower-order deficits significantly contributed to the 3D shape-processing deficit, we investigated whether impairment on 3D tasks remained significant in PCA patients after adjusting for performance on these $2 \mathrm{D}$ and elementary feature tasks. We performed an ANCOVA with performance of the 3D task (errorin-depth) as the dependent variable, group (PCA, healthy controls) as the between-subjects variable, and performance on the lower-order task as the covariate.

\section{Volumetric MRI}

Image acquisition. A high-resolution $\mathrm{T}_{1}$-weighted structural MRI was obtained in patients and healthy controls. In the nine PCA patients, 11 DLBD cases (the two remaining cases had medical contraindications for $\mathrm{MRI}), 14 \mathrm{AD}$ cases, and the 74 controls scanned at the Leuven site, the scans were acquired on a $3 \mathrm{~T}$ Philips Intera system equipped with an eight-channel receive-only head coil (Philips SENSitivity Encoding head coil) using a 3D turbo field echo sequence [coronal inversion recovery prepared 3D gradient-echo images, inversion time (TI) $900 \mathrm{~ms}$, shot interval $=3000 \mathrm{~ms}$, echo time $(\mathrm{TE})=4.6 \mathrm{~ms}$, field of view $(\mathrm{FoV})=$ $250 \times 250 \mathrm{~mm}^{2}$, voxel size $0.98 \times 0.98 \times 1.2 \mathrm{~mm}^{3}$ ]. In the four PCA patients and 44 healthy controls from Liège, a high-resolution $\mathrm{T}_{1^{-}}$ 
A 2D shape from shading

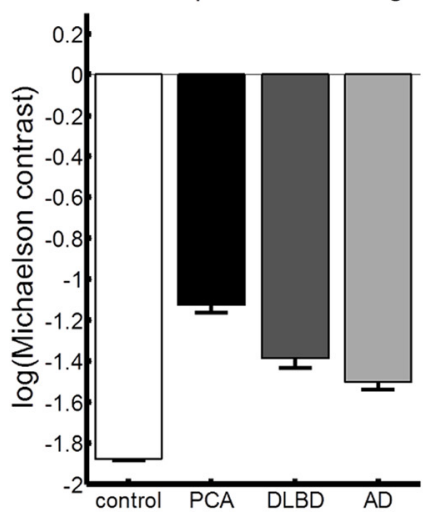

B

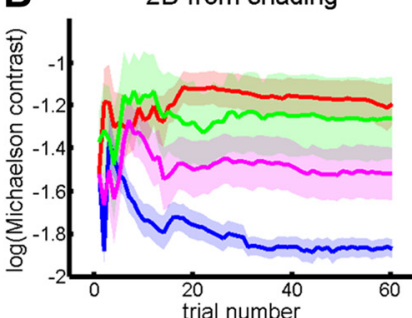

C shading extraction
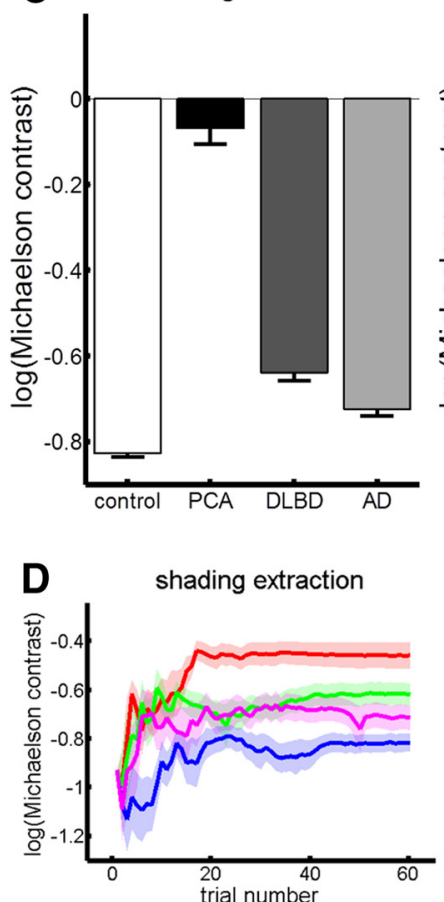

2D shape from texture

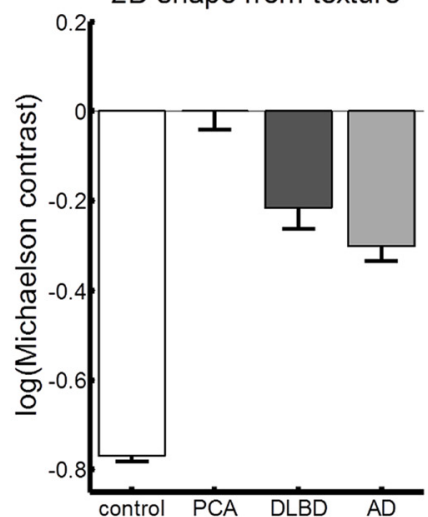

2D from texture

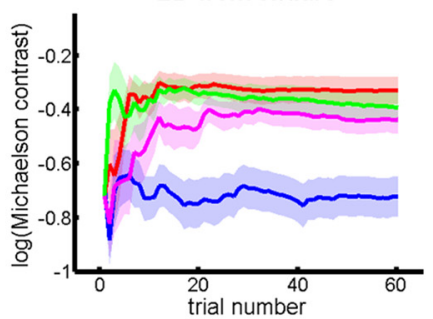

texture extraction
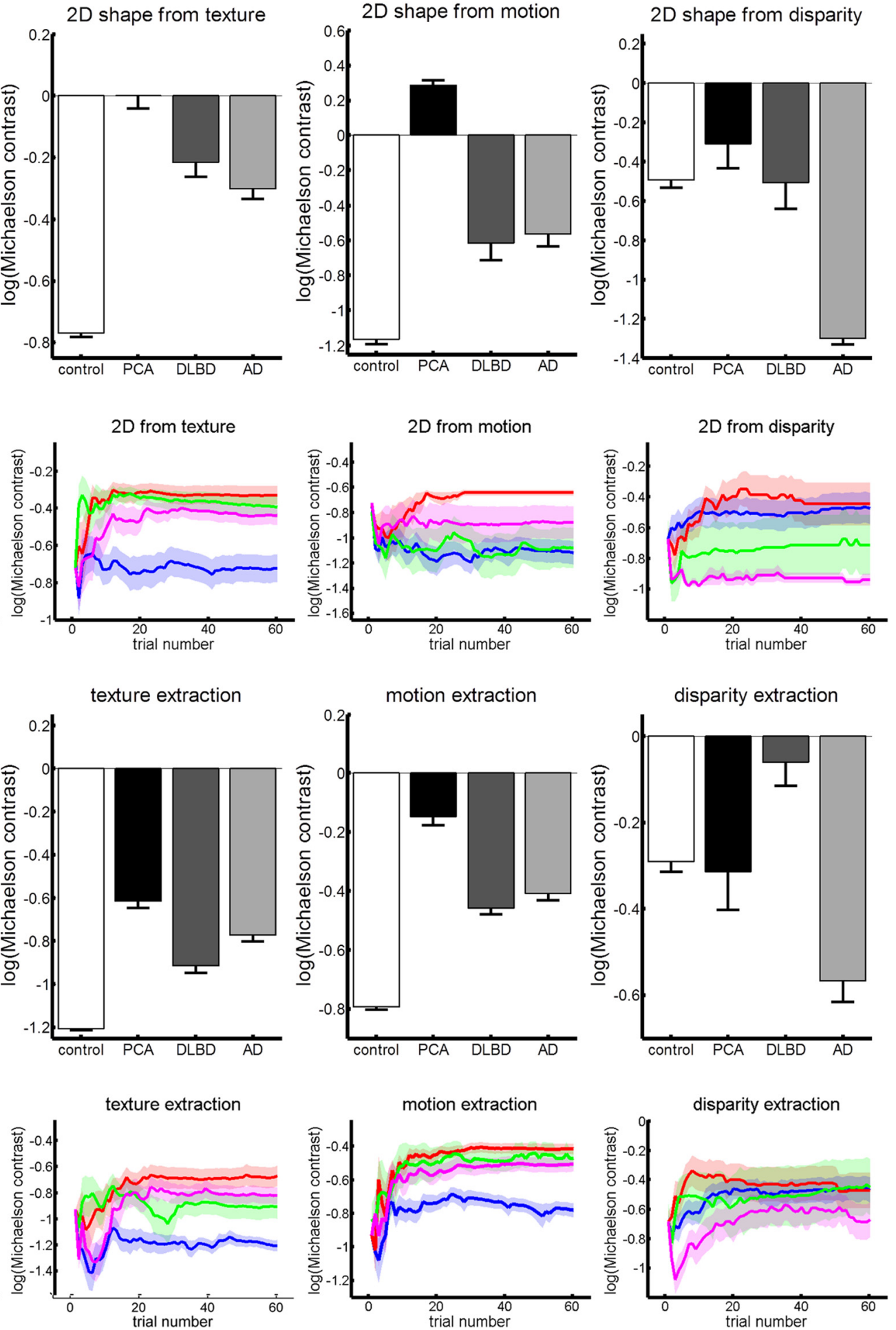

Figure 4. Visuoperceptual deficits on the control tasks. $A, B$, Extraction of $2 D$ shape. $A$, Behavioral performance is expressed as the logarithmic Michaelson contrast between neighboring bars of the gratings to achieve an average performance of $82 \%$. Negative values indicate that the Michaelson contrast is between $0 \%$ and $100 \%$; positive values indicate that the QUEST algorithm estimates the values to be larger than $100 \%$. B. The group-averaged plot of the values obtained during the course of the 60 trials of the staircase procedure. $\boldsymbol{C}-\boldsymbol{D}$, Feature extraction. $\boldsymbol{C}$, Behavioral performance expressed as the logarithmic Michaelson between two squares needed to achieve an average performance of $82 \%$. D, Group-averaged plot of the values obtained during the course of the 60 trials of the staircase procedure. Plots for the staircase procedure: blue, healthy controls; red, PCA patients; green, DLBD patients; magenta, AD patients. Shaded areas reflect SEM.

weighted anatomical image was acquired on a $3 \mathrm{~T}$ head-only scanner (Magnetom Allegra; Siemens) operated with the standard transmitreceive quadrature head coil $\left(\mathrm{T}_{1}\right.$-weighted $3 \mathrm{D}$ magnetization-prepared rapid gradient echo sequence, shot interval $=1960 \mathrm{~ms}$, TI $=1100 \mathrm{~ms}$, $\mathrm{TE}=4.35 \mathrm{~ms}, \mathrm{FoV}=230 \times 173 \mathrm{~mm}^{2}$, voxel size $=0.9 \times 0.9 \times 0.9$ $\mathrm{mm}^{3}$ ). The maximum interval between the structural MRI and the psychophysical experiments was $7 \mathrm{~d}$.

Preprocessing. Preprocessing and statistical analysis were performed with Statistical Parametric Mapping 8 (SPM8, Wellcome Trust Centre for Neuroimaging, London, http://www.fil.ion.ucl.ac.uk/spm) and 
A

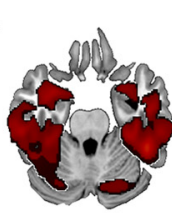

$z=-25$

B

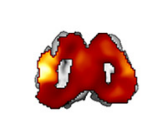

$y=-90$

C

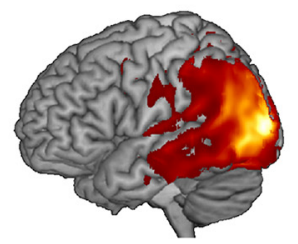

Gray matter atrophy in PCA
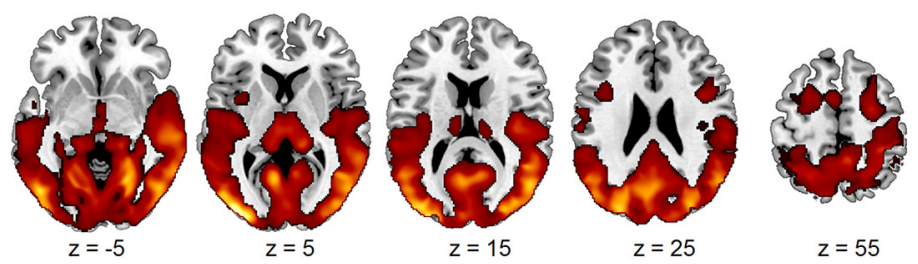

$z=55$
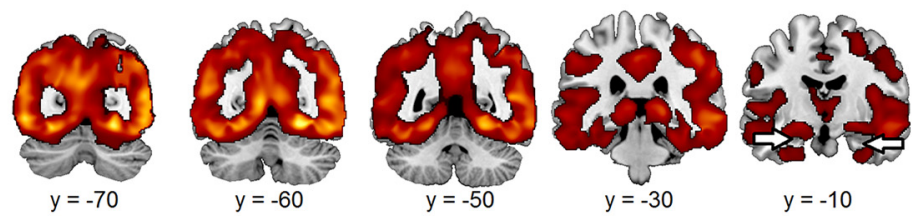

$y=-10$
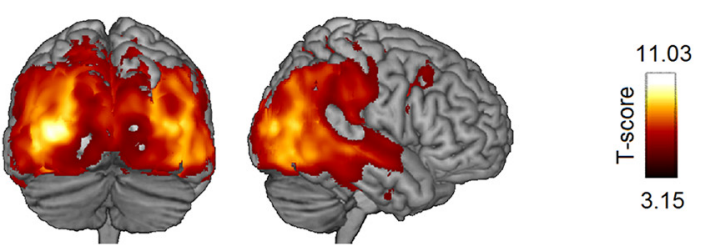

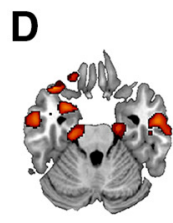

$z=-25$

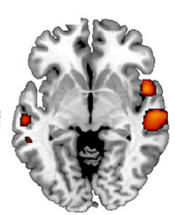

$z=-5$

E
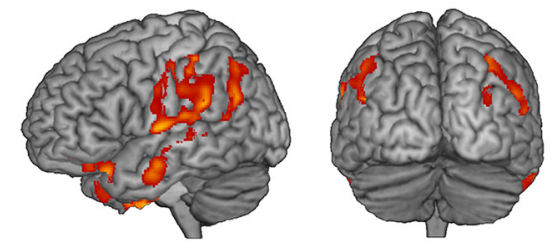

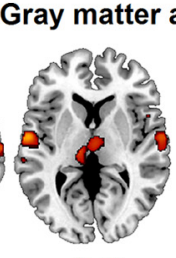

$z=5$

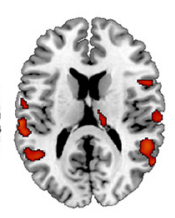

$z=15$

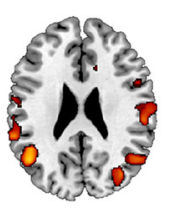

$z=25$

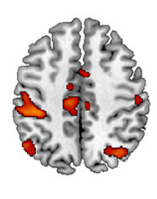

$z=45$
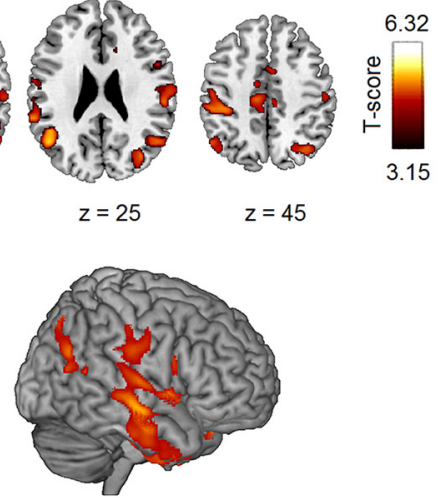

Gray matter atrophy in typical Alzheimer's disease

F

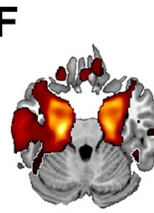

$z=-25$

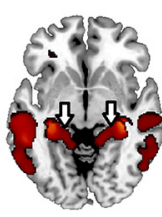

$z=-5$

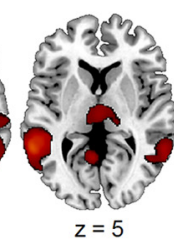

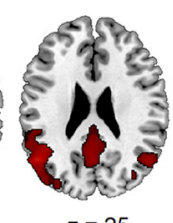

$z=15$
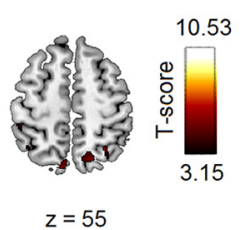

G
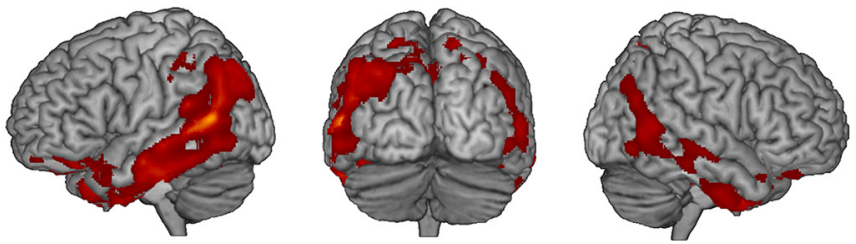

Figure 5. Gray matter volume loss in $\operatorname{PCA}(\boldsymbol{A}-\boldsymbol{C}), \operatorname{DLBD}(\boldsymbol{D}, \boldsymbol{E})$, and typical $\operatorname{AD}(\boldsymbol{F}, \boldsymbol{G})$ versus healthy controls. The $t$ maps indicate the significant reductions of gray matter volume in the different patient groups compared with healthy controls, corrected for age, sex, and scanner effect. All maps are thresholded at cluster-level FWE-corrected $p<0.05$ for a voxel-level threshold of $p<0.001$ uncorrected. $\boldsymbol{A}-\boldsymbol{C}$, Lower gray matter volume in PCA than in healthy control group. The $t$-map is projected onto axial $(\boldsymbol{A})$ and coronal $(\boldsymbol{B})$ slices of a $\mathrm{T}_{1}$-weighted image template available in MRIcron. $\boldsymbol{C}$, Rendering on the lateral surfaces of the brain. $\boldsymbol{D}, \boldsymbol{E}$, Lower gray matter volume is seen in DLBD patients compared with the healthy control group. Shown are axial slices $(\boldsymbol{D})$ and rendering $(\boldsymbol{E})$ on the lateral surfaces of the brain. $\boldsymbol{F}, \mathbf{G}$, Lower gray matter volume is seen in AD patients compared with the healthy control group. Shown are axial slices $(\boldsymbol{F})$ and rendering $(\boldsymbol{G})$ on the lateral surfaces of the brain. The white arrow indicates hippocampal atrophy in AD. the Voxel-Based Morphometry 8 toolbox (VBM8, http://dbm.neuro.uni-jena.de/vbm). High-resolution $\mathrm{T}_{1}$-weighted images were corrected for bias-field inhomogeneities, registered to the Montreal Neurological Institute (MNI) space using linear (12-parameter affine) and nonlinear transformations, and segmented into gray matter, white matter, and CSF within the same generative model (Ashburner and Friston, 2005). Voxel values were modulated for volumetric changes and smoothed with a $8 \times 8 \times 8 \mathrm{~mm}^{3}$ full-width at half-maximum (FWHM) Gaussian kernel.

Voxel-based morphometry. To identify the principal areas of cortical atrophy, we conducted a voxelwise ANCOVA with four groups (PCA, healthy controls, DLBD, and typical $\mathrm{AD})$ as between-subjects factor, and age, sex, and scanner site as nuisance variables using the VBM8 toolbox with the "nonlinear only modulation" option (threshold: cluster-level FWEcorrected $p<0.05$ for a voxel-level threshold of $p<0.001$ uncorrected; Poline et al., 1997).

To investigate the correlation between regional gray matter volume loss and $3 \mathrm{D}$ shape processing, individual error-in-depth scores of the 13 PCA patients on the 3D shape-processing tasks were entered as independent variables in voxelwise linear regression analyses with modulated gray matter volume as the dependent variable. The threshold for significance was set to a cluster-level FWE-corrected $p<0.05$ for a voxel-level threshold of uncorrected $p<0.005$ (Poline et al., 1997).

We conducted a similar voxelwise linear regression analysis in the patient control groups and evaluated the overlap between the significance map obtained in PCA at the preset threshold (see above) and that obtained in the patient control groups. When assessing the overlap, the latter maps were thresholded at voxelwise uncorrected $p<0.01$ because we mainly wanted to determine how specific the correlation was for PCA.

We assessed the effect of scanner site in a voxelwise analysis of covariance with scanner site as covariate of interest, age, and sex as covariates of no interest and the modulated gray matter images from the 118 healthy controls as dependent variables. The threshold for significance was set to a cluster-level FWE-corrected $p<0.05$ for a voxel-level threshold of uncorrected $p<0.005$.

\section{fMRI}

To delineate the functional anatomy network in healthy controls, we conducted an fMRI study in 18 cognitively intact older adults for 3D shape-from-shading and 3D shape-frommotion using appropriate 2D control stimuli (see Fig. 11A). We only selected monocular cues for fMRI for practical reasons and left out texture as a cue because of the number of control conditions needed for 3D shape-fromtexture (Georgieva et al., 2008).

The fMRI substudy was only offered to those PCA patients who underwent scanning at the Leuven site $(n=9)$ to avoid between-scanner effects on the BOLD signal. Cases 1, 2, 6, 7, and 
Lower gray matter matter volume in PCA compared to DLBD

A
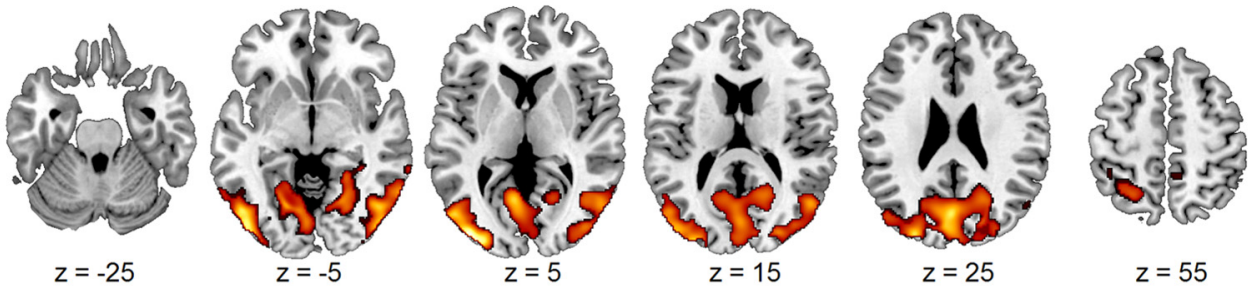

B
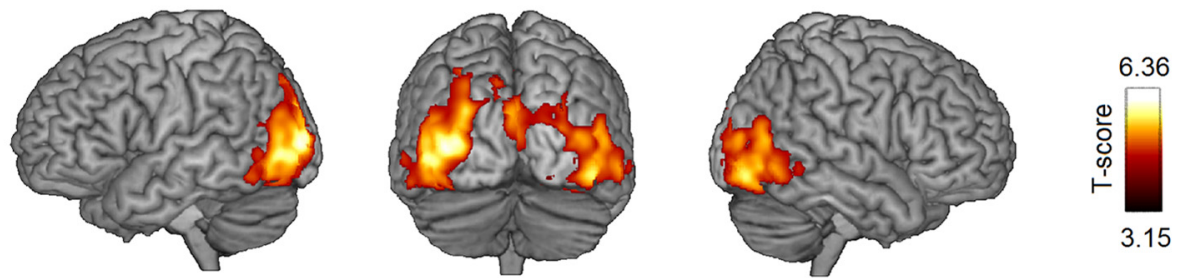

Lower gray matter volume in PCA compared to typical Alzheimer's disease

C
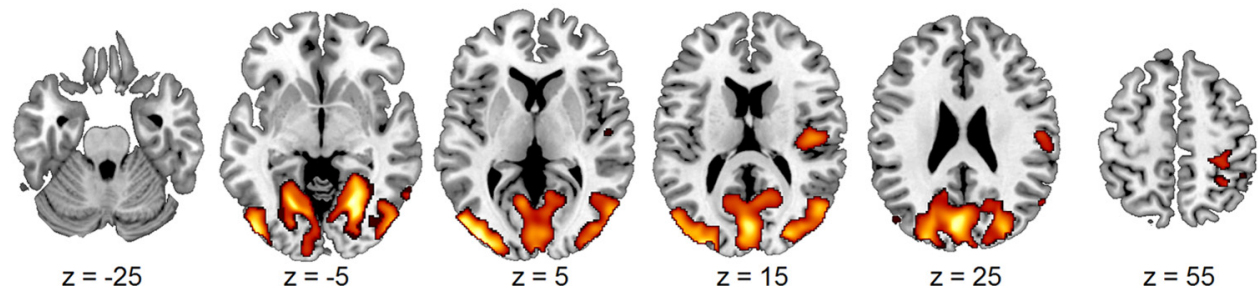

D
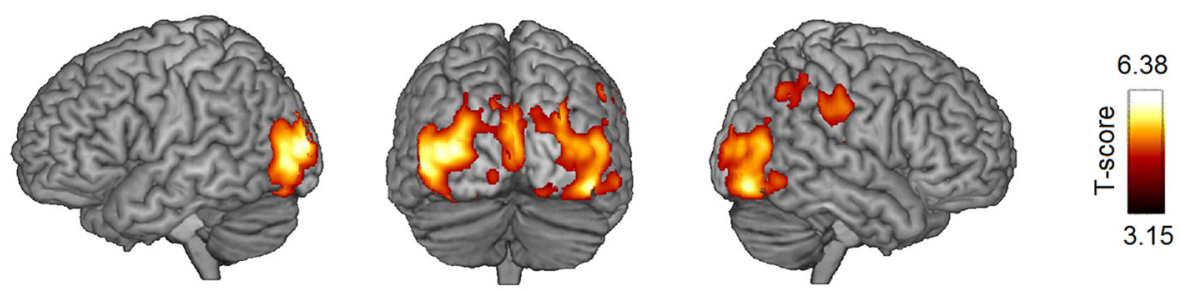

E

\section{Lower gray matter volume in AD compared to PCA}

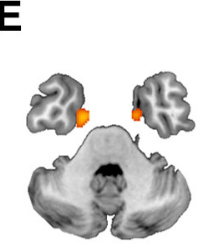

$z=-36$

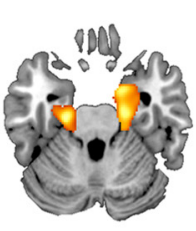

$z=-26$

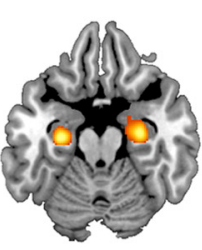

$z=-20$

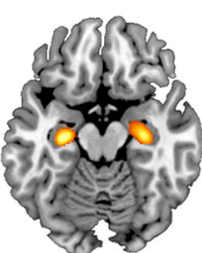

$z=-16$

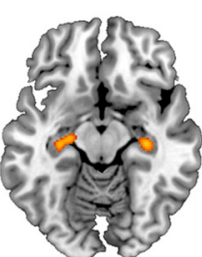

$z=-12$

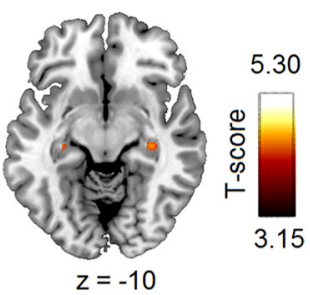

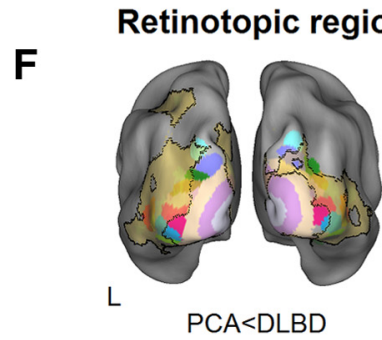
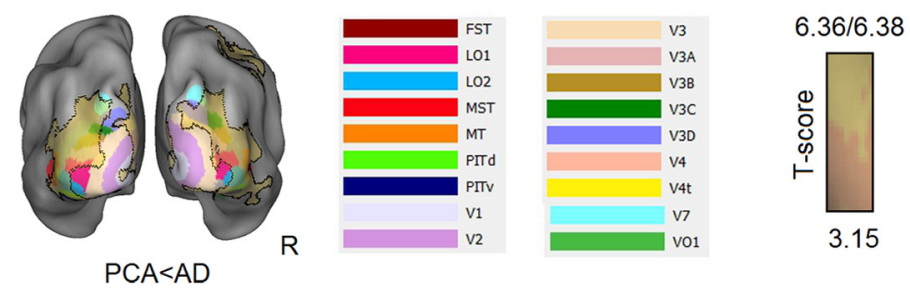

Figure 6. Gray matter volume loss in PCA versus patient control groups. The $t$ maps indicate the significant differences between patient groups, corrected for age, sex, and scanner effect. All maps are thresholded at cluster-level FWE-corrected $p<0.05$ for a voxel-level threshold of $p<0.001$ uncorrected. $\boldsymbol{A}, \boldsymbol{B}$, Lower gray matter volume can be seen in PCA patients compared with the DLBD group. Shown are axial slices $(\boldsymbol{A})$ and rendering $(\boldsymbol{B})$ on the lateral surfaces of the brain. $\boldsymbol{C}, \boldsymbol{D}$, Lower gray matter volume can be seen in PCA patients compared with the $A D$ group. Shown are axial slices $(\boldsymbol{C})$ and rendering $(\boldsymbol{D})$ on the lateral surfaces of the brain. $\boldsymbol{E}$, Lower gray matter volume can be seen in AD patients compared with the PCA group (axial slices). $\boldsymbol{F}, \mathrm{T}$-maps for the contrasts of PCA minus DLBD and PCA minus AD are overlaid onto a probabilistic retinotopic map, indicating the differential volume loss in extrastriate course in PCA compared with the two patient control groups. 


\section{D shape-from-shading cluster in PCA patients}

A
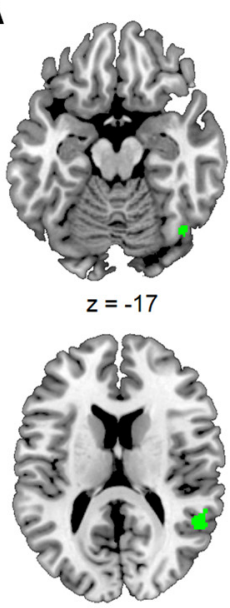

$z=14$
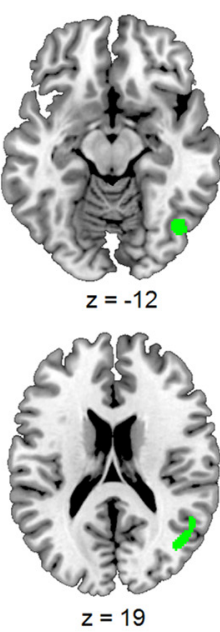

$z=19$
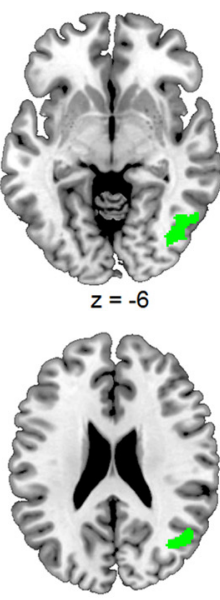

$z=24$
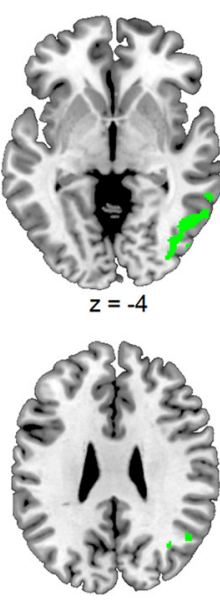

$z=28$
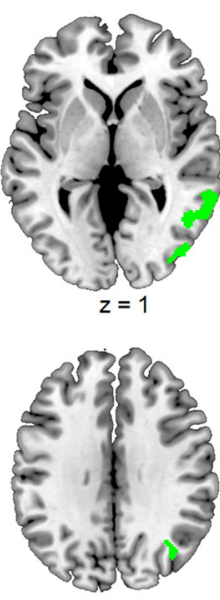

$z=32$
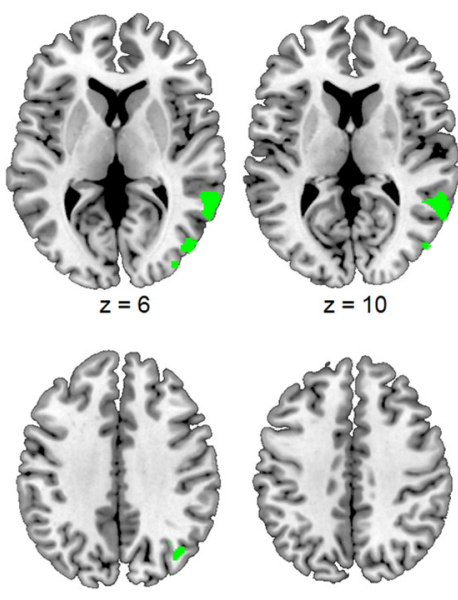

$z=37$

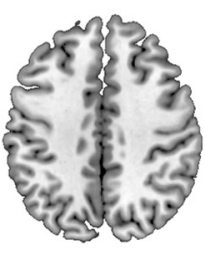

$z=42$
B

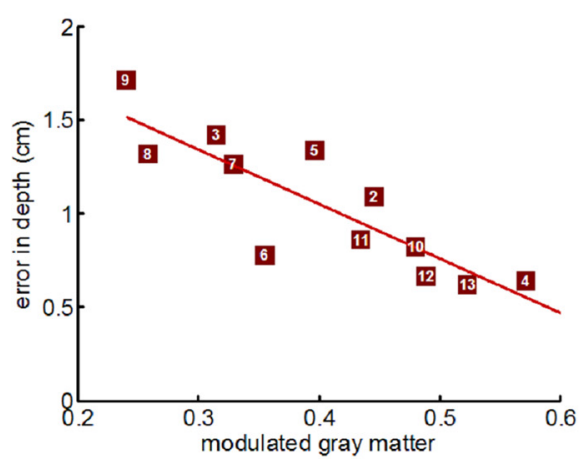

C 3D structure from shading and affected retinotopic regions

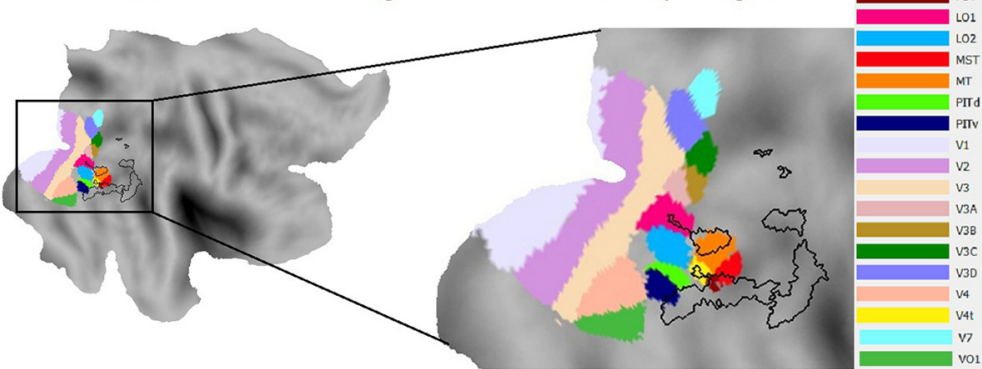

Figure 7. Correlation between gray matter volume loss in PCA and 3D shape-from-shading. $A$, Voxel-based correlation of gray matter volume with performance of PCA patients on the 3D shape-from-shading task (voxel-level uncorrected $p<0.005$; cluster-level FWE-corrected $p<0.05$ ). $\boldsymbol{B}$, Scatterplot of mean gray matter volume in the inferior temporal cluster and performance on the 3D shape-from-shading task illustrating the distribution of data points obtained in this region with the volumetric regression analysis. Red squares, PCA patients (case numbers correspond to those used in Table 1); red line, regression line obtained in PCA patients. C, Probabilistic retinotopic map (Abdollahi et al., 2014) and 3D shape-from-shading cluster (black line) thresholded at a cluster-level corrected $p<0.05$ for an uncorrected voxel-level $p<0.005$.

12 participated and the remaining 4 subjects refused, principally for the following practical reasons: to avoid an extra visit, long-distance travel, or additional stress for patient or because of unwillingness to lie still in the MRI scanner for $1.5 \mathrm{~h}$.

Experimental design. Visual stimuli were projected from a liquid crystal display projector (Barco Reality 6400im $1024 \times 768$, $75 \mathrm{~Hz}$ refresh frequency) onto a translucent screen positioned in the bore of the magnet at a distance of $36 \mathrm{~cm}$ from the point of observation. Participants viewed the stimuli through a tilted mirror attached to the head coil and wore an eye patch over the right eye. Participants were asked to maintain fixation on a small red target $\left(0.45^{\circ} \times 0.45^{\circ}\right)$ in the center of the screen. Fixation was monitored online using an MR-compatible ASL eye tracking system 5000 (Applied Science Laboratories).

The 3D shape-from-shading condition was identical to those used by Georgieva et al. (2008). In the two control conditions (see Fig. 11A), we eliminated appearance of depth by randomly repositioning the pixels within the boundary of each object (2D pixel-scrambled) or by mimicking the pattern of shading gradients with three to five randomly shaped ovals with blurred edges on a light background (2D shaded-blobs) (Georgieva et al., 2008).

The fMRI experiment used a block design with 20 s epochs in which we presented 15 stimuli for $1333 \mathrm{~ms}$ without gaps. Conditions were presented in different epochs and repeated three times in a time series. Six time series were recorded in each participant and presentation order of the conditions was randomized between the six time series. The fMRI experiment also contained three further conditions related to 3D shapefrom-motion and two control conditions, but for the sake of brevity these results will not be reported here.

Image acquisition and analysis. The whole-brain functional scans consisted of $\mathrm{T}_{2}^{*}$-weighted gradient-echo echoplanar images obtained on a $3 \mathrm{~T}$ Philips Intera scanner equipped with an 8-channel receive-only head coil ( 6 time series, 212 scans per time series, repetition time $=2000 \mathrm{~ms}$, TE $=$ $30 \mathrm{~ms}, 2.75 \times 2.75 \mathrm{~mm}^{2}$ in-plane resolution, $363.75 \mathrm{~mm}$ thick axial slices without gap). The $\mathrm{T}_{2}^{*}$-weighted images were realigned and coregistered to the $\mathrm{T}_{1}$-weighted image. The $\mathrm{T}_{1}$-weighted image was warped into the MNI space using the procedures implemented in the VBM8 toolbox (see above) and the resulting deformation field was used to spatially normalize the functional images. The voxel size of the functional images in MNI space was $3 \times 3 \times 3 \mathrm{~mm}^{3}$. The images were spatially smoothed with a $6 \times$ $6 \times 6 \mathrm{~mm}^{3}$ FWHM Gaussian kernel before statistical analysis.

We examined in each PCA patient whether his/her activity pattern in the 3D shape-from-shading network differed from that seen in the control group, controlling for the effect of regional gray matter volume. To this end, we determined the effect size for contrast 1 (3D shape-fromshading minus $2 \mathrm{D}$ pixel-scrambled) and 2 (3D shape-from-shading minus $2 \mathrm{D}$ shaded-blobs) in the different clusters of the 3D shape-fromshading network. For each control, this network was defined using a random-effects analysis (conjunction of contrasts 1-2) in the remaining 17 healthy controls (Georgieva et al., 2008). With this leave-one-out approach we avoided a bias in favor of the control group that might arise 


\section{D shape-from-disparity cluster in PCA patients}

A

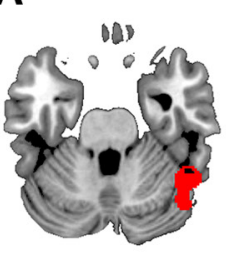

$z=-27$

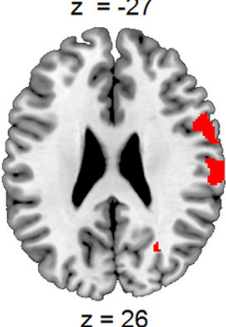

$z=26$

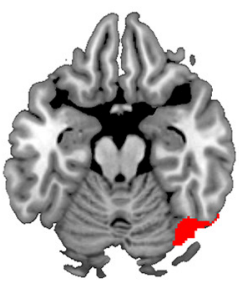

$z=-20$

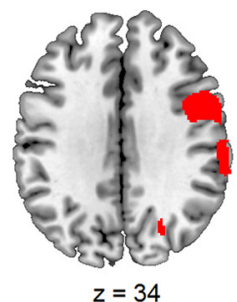

$z=34$

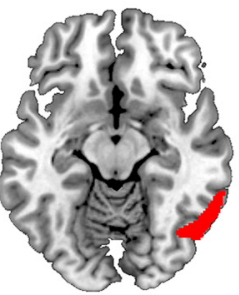

$z=-12$

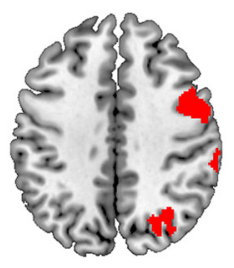

$z=40$

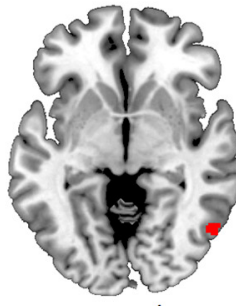

$z=-4$

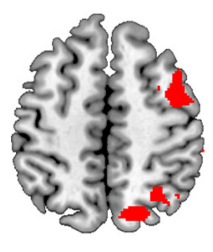

$z=51$
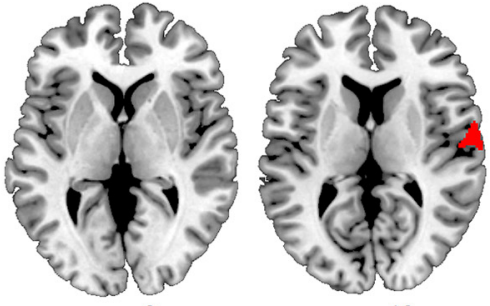

$z=10$
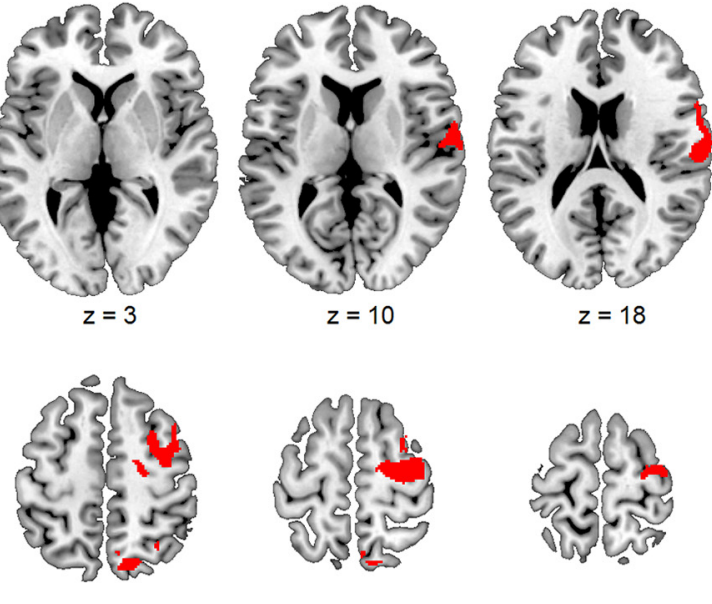

$z=56$

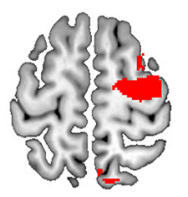

$z=63$

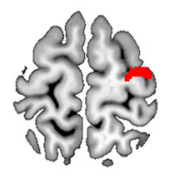

$z=68$
B

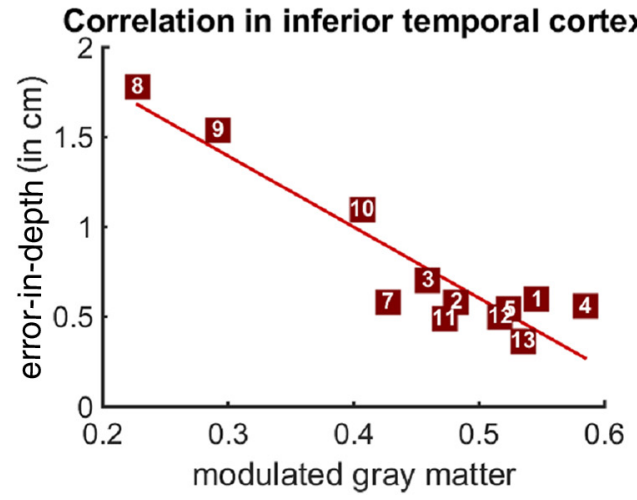

C

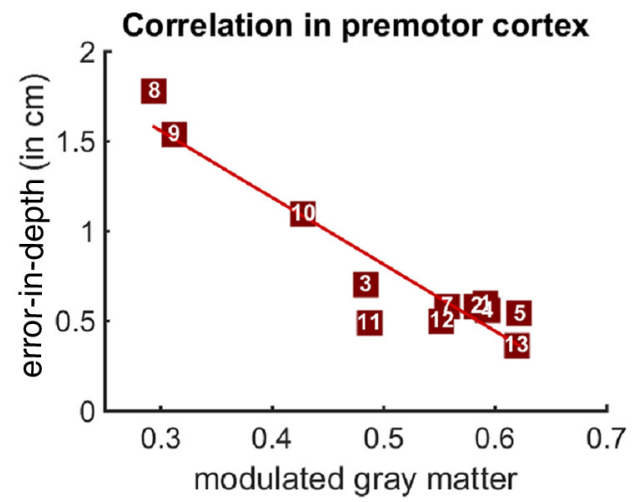

Figure 8. Correlation between gray matter volume loss in PCA and 3D shape-from-disparity. $A$, Voxel-based correlation of gray matter volume with performance of PCA patients on the 3D shape-from-disparity task (voxel-level uncorrected $p<0.005$; cluster-level FWE-corrected $p<0.05$ ). $\boldsymbol{B}$, Scatterplot of mean gray matter volume in the inferior temporal cluster and performance on the 3D shape-from-disparity task. C, Scatterplot of mean gray matter volume in the right premotor cluster and performance on the 3D shape-from-disparity task. Red squares, PCA patients; red lines, regression lines obtained in PCA patients.

had we defined the network in the control group and examined activity levels in patients in that predefined network (Gillebert et al., 2011). For each PCA patient, the effect size for the two contrasts in the 3D shapefrom-shading network was compared with that obtained in healthy controls using regional gray matter volume as a covariate of no interest (Crawford et al., 2011) (threshold: two-tailed $p<0.05$ ).

\section{Results}

The primary purpose of this study was to determine whether and for which cues $3 \mathrm{D}$ shape processing was affected in PCA, whether this could be explained by deficits at lower levels of processing, and which regions were implicated structurally. Furthermore, we overlaid the fMRI activity pattern for $3 \mathrm{D}$ shape processing in healthy controls directly with the volumetric changes that correlate with $3 \mathrm{D}$ shape processing in PCA and also evaluated how functional activity levels in the 3D shape network were altered in a subset of PCA patients. Finally, we also evaluated how disease specific the behavioral deficits and the correlations with gray matter volume were by comparing our findings to two patient control groups, DLBD and typical AD.

Behavioral comparison between PCA and controls $3 D$ shape perception

The error-in-depth for the four cues and the four groups is shown in Figure 2. Repeated-measures ANOVA $(4 \times 4$ design $)$ with group (healthy controls, PCA, DLBD, and AD patients) and cue (shading, texture, motion, and binocular disparity) as factors and error-in-depth as an dependent variable revealed a significant main effect of group $\left(F_{(3,65)}=17.09, p<0.001\right)$ and of cue $\left(F_{(2.18,141.42)}=7.44, \mathrm{G}-\mathrm{G}\right.$ adjusted $\left.p=0.001\right)$ and a significant group-by-cue interaction $\left(F_{(6.53,141.42)}=3.29, \mathrm{G}-\mathrm{G}\right.$ adjusted $p=$ 0.004; Fig. 2, Table 2). According to post hoc comparisons, errorin-depth was larger in PCA than in healthy controls $(p<0.001)$ for each of the monocular cues. Error-in-depth tended to be larger in PCA than in AD for texture $(p=0.005)$. Error-indepth did not differ between PCA and DLBD for any of the cues ( $p>0.31$; Fig. 2 ), nor was there any difference between PCA and any of the control groups for binocular disparity ( $p \geq 0.28$; Fig. 2).

The average ellipse parameters representing the in-plane distribution of the responses of PCA and normal subjects are shown in Figure $3 C$ for each of the cues. In healthy controls, the angle and elongation is very similar between cues because the underlying 3D structure is the same. The ellipses in PCA are systematically larger and wider than those of healthy controls. The main parameters of these ellipses are shown in Figure 3, D-G. According to the repeated-measures ANOVA with group and cue as factors, there was a significant main effect of group on all 4 ellipse 
A

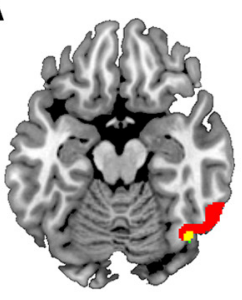

$z=-17$

\section{Overlap between 3D shape-from-shading and 3D shape-from-disparity}

B

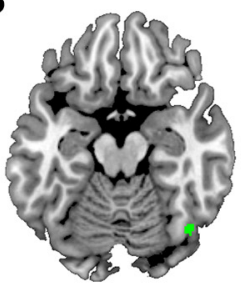

$z=-17$

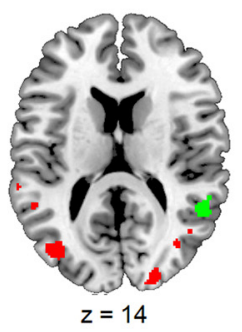

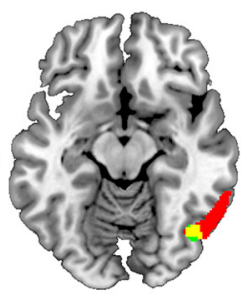

$z=-12$

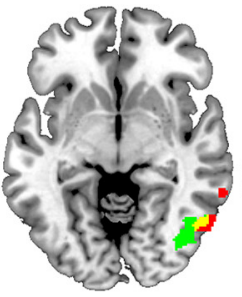

$z=-6$

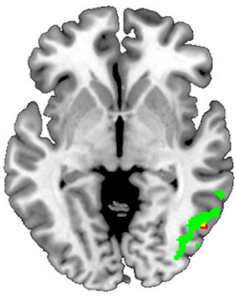

$z=-4$

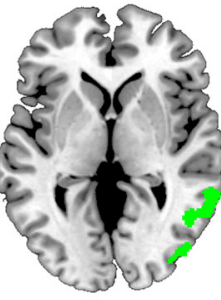

$z=1$

3D shape-from-shading

3D shape-from-disparity

overlap

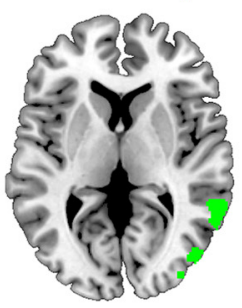

$z=6$

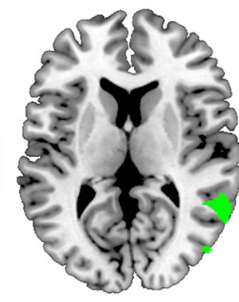

$z=10$

Overlap between 3D shape-from-shading and 2D shape-from-shading

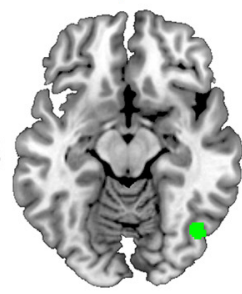

$z=-12$

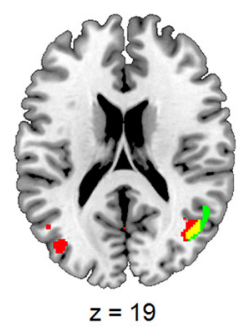

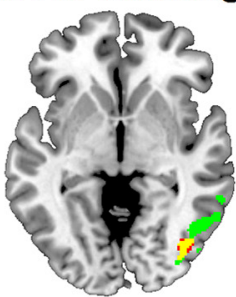

$z=-4$

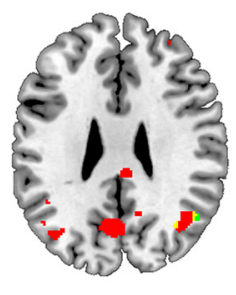

$z=28$

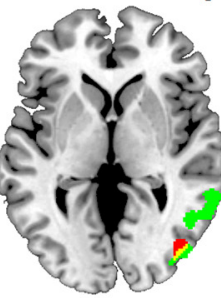

$z=1$

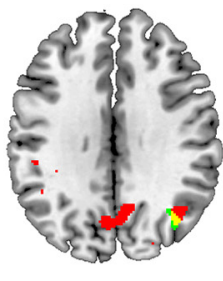

$z=32$

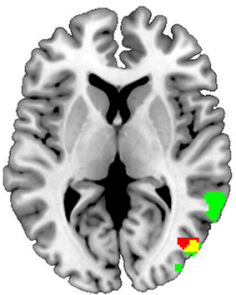

$z=6$

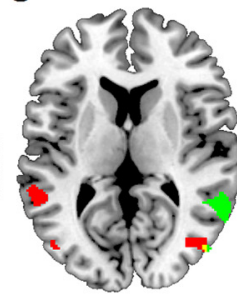

$z=10$
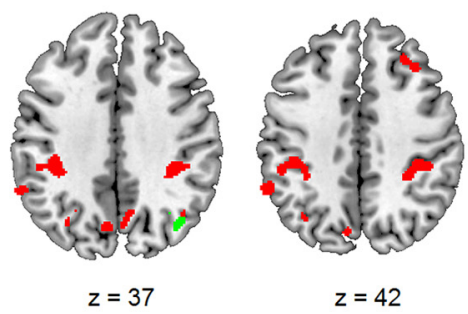

$z=42$

Figure 9. A, 0verlap between the cluster shown in Figure $7 A$ and the cluster shown in Figure $8 A$. B, Overlap between the cluster shown in Figure $7 A$ and the map of the correlation between volume and 2D orientation discrimination. Green, Cluster where gray matter volume correlates with 3D shape-from-shading (voxel-level uncorrected $p<0.005$ and cluster-level FWE-corrected $p<0.05$ ); red, map of correlation of gray matter volume with 3D shape-from-disparity $(\boldsymbol{A})$ and 2D-from-shading $(\boldsymbol{B})$ in PCA patients (voxel-level uncorrected $p<0.005$ ); yellow, overlap.

parameters (G-G adjusted $p \leq 0.01$; Fig. $3 C-G$ ), a main effect of cue on location $(x$ and $y)$ and on size $(p \leq 0.002)$, and a groupby-cue interaction on location ( $y$-coordinate, $p=0.006$; Fig. $3 D-$ $G)$. According to post hoc comparisons, ellipse size differed significantly between PCA and healthy controls for all 4 cues ( $p \leq$ 0.003). The location of the ellipse center differed between PCA and healthy controls for shading $(y$-coordinate $p=0.004)$ and for texture ( $y$-coordinate $p=0.003$ ). No other differences between PCA and the patient groups reached our stringent corrected significance threshold.

In PCA, error-in-depth and in-plane distribution of responses differed from random for all cues $(p \leq 0.01)$ except texture ( $p=$ 0.48 ; Fig. 2). Performance of 3D shape-from-shading, motion, and disparity in PCA also differed from a distribution based on random motor control errors for shading, motion, and disparity $(p<0.05$ in $>99 \%$ iterations; Fig. 2).

At an individual level (Table 2), all PCA patients except Case 4 performed worse than healthy controls $(p<0.05$; Crawford and Garthwaite, 2002) on 3D shape processing for monocular cues, with 8 patients performing worse than healthy controls for all 3 monocular cues. Two of the PCA patients were significantly impaired on the 3D shape-from-disparity task (Table 2). There were a number of dissociations between 3D tasks in PCA patients: 3D shape-from-motion was significantly more affected than 3D shape-from-disparity or 3D shape-from-shading in 9 and 5 cases, respectively. 3D shape-from-texture was significantly more affected than 3D shape-from-disparity and 3D shape-fromshading in 8 and 2 cases, respectively. 3D shape-from-shading was significantly more affected than 3D shape-from-disparity in 5 cases.

The AUC of error-in-depth for discriminating between PCA and controls was 0.97 for texture, 0.94 for shading, 0.93 for motion, and 0.70 for disparity. For PCA compared with AD patients, the AUC was 0.81 for texture, 0.69 for motion, 0.60 for shading, and 0.54 for disparity. The AUC of error-in-depth for discriminating between PCA and DLBD patients was 0.58 for texture, 0.45 for shading, 0.54 for motion, and 0.51 for disparity.

\section{$2 D$ orientation discrimination}

We investigated whether the $2 \mathrm{D}$ processing level was also affected (Figs. 1D, $4 A, B$ ). However, we were unable to obtain reliable thresholds for the disparity cue in four of the PCA cases (Table 2) and, because of these missing data, we analyzed performance for the monocular cues separately from that for the binocular cue.

For the monocular cues, a $4 \times 3$ ANOVA analysis revealed main effects of group $\left(F_{(3,59)}=17.64, p<0.001\right)$ and of cue $\left(F_{(1.53,90.4)}=112.52\right.$, G-G adjusted $\left.p<0.001\right)$, as well as a significant group-by-cue interaction $\left(F_{(4.6,90.4)}=2.53, \mathrm{G}-\mathrm{G}\right.$ adjusted $p=0.039$; Fig. $4 A$, Table 2). PCA patients needed a larger orientation difference than healthy controls to reach criterion for all 
monocular cues $(p<0.001)$. For motion, they needed a larger orientation difference than $\mathrm{AD}(p=0.005$; Fig. $4 A)$. No other differences were found between PCA and the patient control groups ( $p>$ 0.06).

No differences between groups were found for binocular disparity $\left(F_{(3,52)}=\right.$ $2.40, p=0.08$ ).

\section{Elementary feature extraction}

We also evaluated whether elementary feature extraction was affected (Figs. 1E, $4 C, D)$. However, we were unable to obtain reliable thresholds for the disparity cue in five of the PCA cases (Table 2) and therefore analyzed performance for the monocular cues separately from that for the binocular cue.

A $4 \times 3$ ANOVA revealed significant main effects of group $\left(F_{(3,62)}=24.7, p<\right.$ $0.001)$ and of cue $\left(F_{(2,124)}=36.85, p<\right.$ 0.001 ), as well as a significant group-bycue interaction $\left(F_{(6,124)}=3.48, p=0.003\right.$; Fig. $4 C$, Table 2). To reach criterion, PCA patients needed a larger contrast than healthy controls $(p<0.001)$ regardless of cue type (Fig. 4C). They also needed a significantly larger contrast than DLBD and $\mathrm{AD}$ patients for luminance ( $p=0.001$; all others $p>0.02$ ).

No differences between groups were found for binocular disparity $\left(F_{(3,56)}=\right.$ $1.93, p=0.14)$.

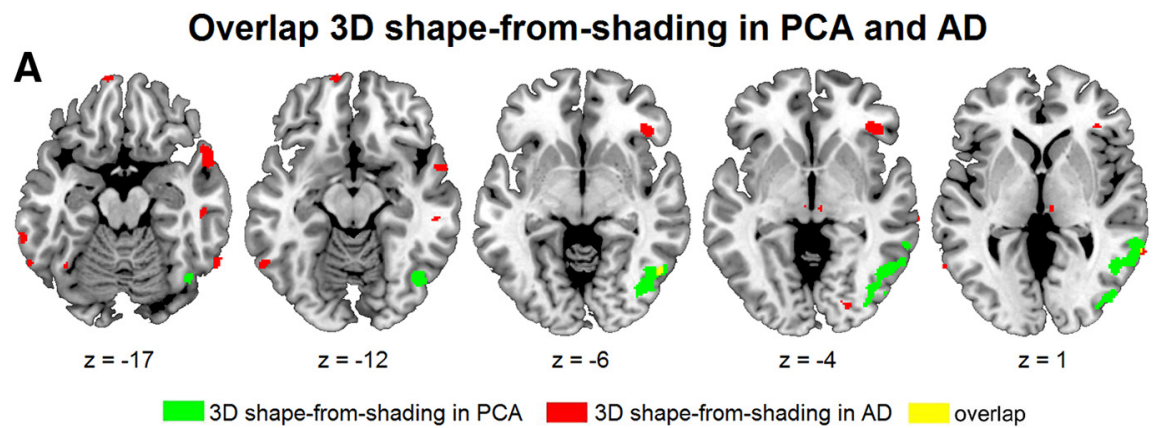

B

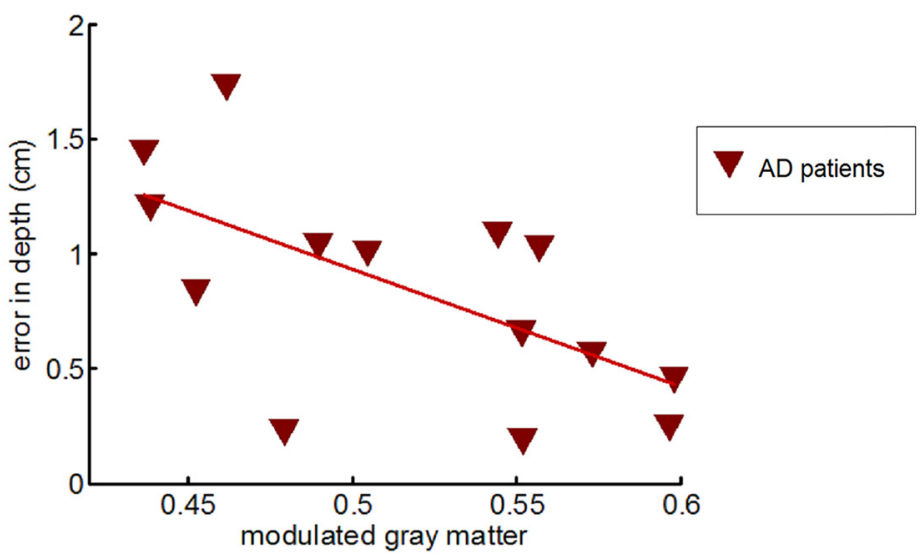

Figure 10. Overlap between the cluster shown in Figure 7A obtained in PCA patients for 3D shape-from-shading and a map of the correlation between volume and 3D shape-from-shading in AD patients. $A$, Green, Cluster where gray matter volume correlates with 3D shape-from-shading (voxel-level uncorrected $p<0.001$ and cluster-level FWE-corrected $p<0.05$ ); red, map of correlation of gray matter volume with 3D shape-from-disparity in $A D$ (voxel-level uncorrected $p<0.01$; without cluster-level FWEcorrection); yellow, overlap. $\boldsymbol{B}$, Scatterplot of mean gray matter volume obtained in the region shown in $\boldsymbol{A}$ and performance on the $3 D$ shape-from-disparity in AD patients. Red triangles, AD patients; red line, regression line obtained in AD patients $(r=-0.626)$.
Relationship between $3 D$ and lower-order deficits

We investigated whether the deficits for $2 \mathrm{D}$-level processing or elementary feature extraction could account for the 3D shapeprocessing deficit. According to a stepwise multiple linear regression analysis, performance of PCA patients on the 3D shape-fromshading task could be partly predicted by performance at the $2 \mathrm{D}$ processing level $\left(R^{2}\right.$ adjusted $\left.=0.46, p=0.007\right)$. Performance on the $3 \mathrm{D}$ shape-from-texture task could also be partly explained by performance at the elementary feature extraction level $\left(R^{2}\right.$ adjusted $=0.28, p=0.037$ ). Performance on the 3D shape-frommotion and 3D-shape-from-disparity task could not be predicted by performance on the lower-order tasks $(p>0.1)$.

When performance at the $2 \mathrm{D}$-processing level was included as covariate in a one-way ANOVA with group (healthy controls, PCA patients) as the factor and performance on the 3D shapefrom-shading task as the dependent variable, the effect of group remained significant $\left(F_{(1,36)}=6.99, p=0.01\right)$. The difference in performance between healthy controls and PCA patients on the 3D shape-from-texture task also remained significant when performance at the elementary feature extraction level was included as the covariate of no interest $\left(F_{(1,36)}=14.39, p<0.001\right)$.

\section{Volumetric comparison between PCA patients and control groups}

The volumetric comparison of the PCA group with the controls confirmed the predominantly posterior localization of the atrophy (Fig. 5A-C). In the group analysis, primary visual cortex was relatively spared, at least the part representing central vision, whereas lateral occipital and medial occipital cortex were involved together with occipitotemporal, occipitoparietal, and posterior parietal cortex. Dorsal premotor cortex was also atrophic (Fig. 5A-C). Medial pulvinar and lateral geniculate nuclei also exhibited significant volume loss (Fig. 5A-C).

Compared with DLBD and AD, extrastriate cortex showed significant volume loss in PCA (Fig. $6 A-D, F$ ). Inversely, the anterior portion of the hippocampal formation was atrophic in $\mathrm{AD}$ compared with PCA (Fig. 6E). We did not find any regions of greater volume loss in DLBD compared with PCA.

Relationship between volume loss and 3D-processing deficits In PCA, scores on the 3D shape-from-shading task correlated significantly with gray matter volume loss in right posterior inferior temporal cortex extending into lateral temporal and inferior parietal cortex (extent total cluster $23941.5 \times 1.5 \times 1.5 \mathrm{~mm}^{3}$ voxels, cluster-level corrected $p=0.010$; Fig. 7 ). The highestsignificance peak was in the right inferior temporal gyrus (ITG; $44,-63,-6, z=3.71)$, followed by a peak in the right inferior occipital gyrus $(36,-72,-5, z=3.59)$ and a peak in the right middle temporal gyrus $(63,-45,3, z=3.33)$. The correlation between $3 \mathrm{D}$-from-shading and right inferior temporal volume loss was also present when we excluded the monocularly blind Case 6 (extent total cluster 8414 cluster-level $p<0.001, r=$ -0.96).

We also obtained a significant correlation between volume loss and 3D shape-from-disparity in PCA in right inferior temporal cortex $(50,-51,-27, z=4.20$, extent 1958 , corrected $p=$ 0.017; Fig. $8 A, B)$ and right premotor cortex $(44,9,34, z=4.58$, 
A fMRI study: shading stimuli

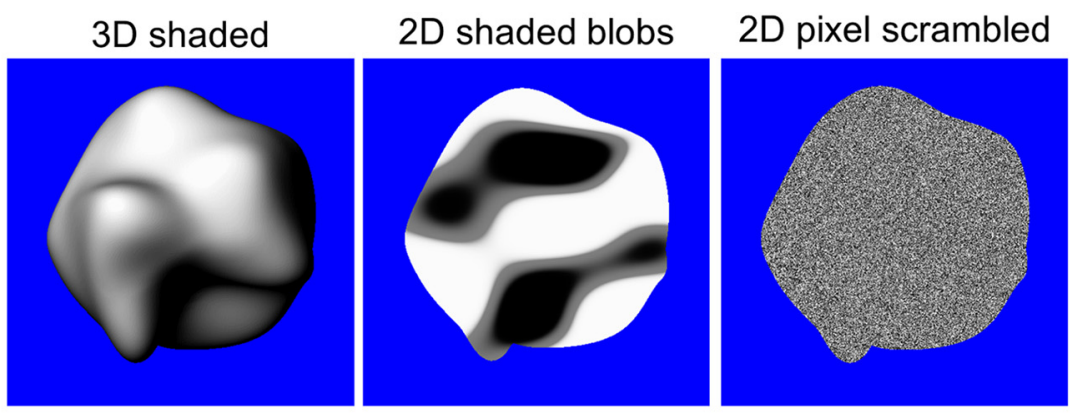

B

3D shape-from shading network

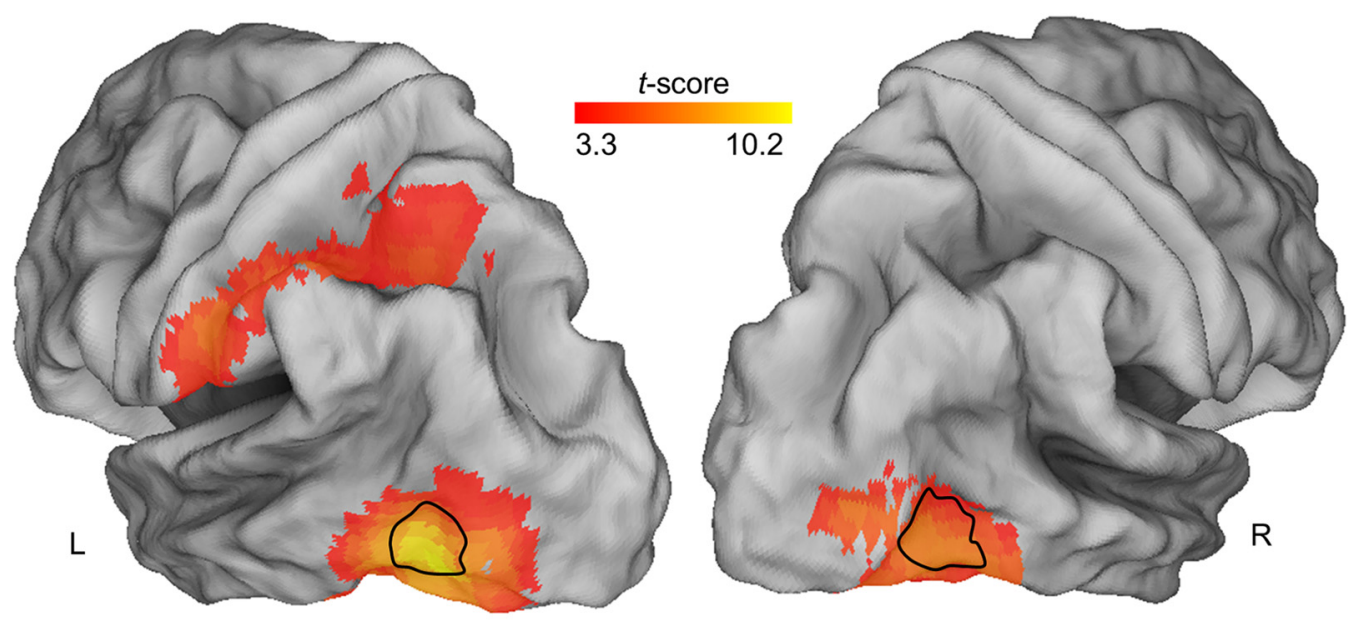

C

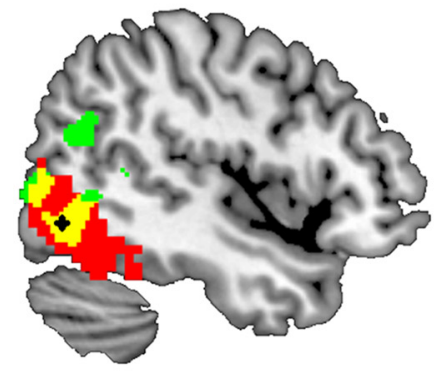

$x=46$

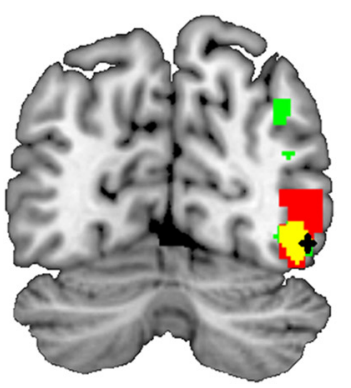

$y=-70$

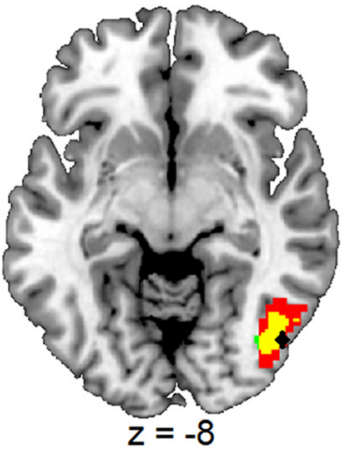

overlap right ITG

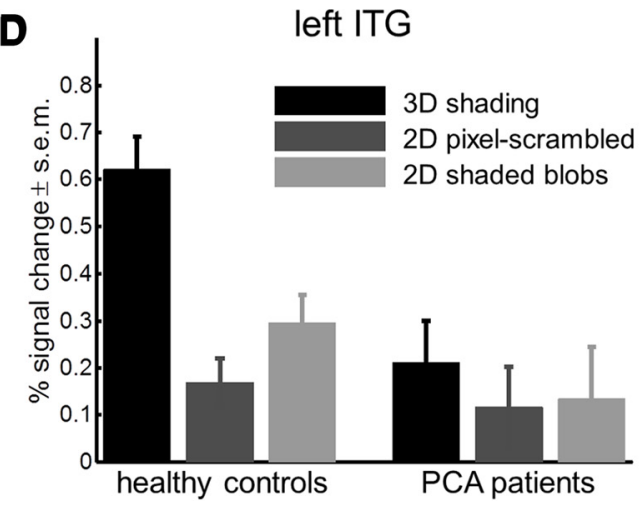

E

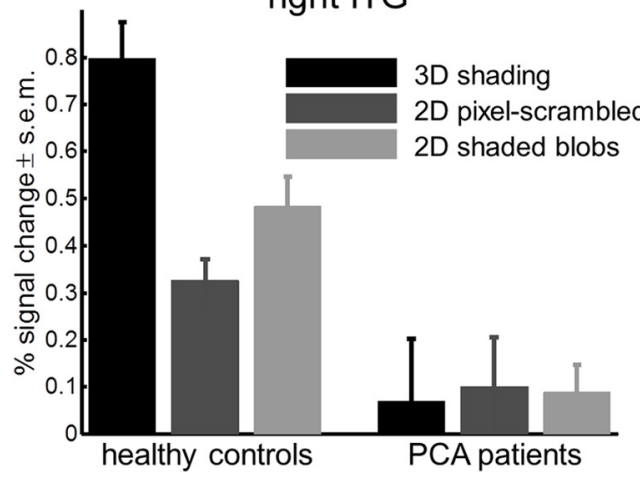


extent 5558, corrected $p<0.001$; Fig. $8 A, C)$. The inferior temporal $3 \mathrm{D}$ shape-from-disparity cluster lay anterior to the $3 \mathrm{D}$ shape-from-shading cluster and there was some overlap between the right occipitotemporal cluster obtained for 3D shape-fromshading $(0.08 \%$ of the total cluster extent) at our preset threshold and that obtained for $3 \mathrm{D}$ shape-from-disparity $(0.10 \%$ of the total cluster extent; Fig. 9A, yellow).

A whole-brain analysis did not reveal significant correlations between volume loss and 3D shape processing for motion and texture. Even if we lowered the threshold for these maps to voxellevel $p<0.01$, we did not find any overlap between the right posterior inferior temporal cluster obtained for shading and disparity and 3D shape-from-motion or 3D shape-from-texture.

We overlaid the inferior temporal cluster obtained for 3D shape-from-shading with a probabilistic retinotopic map (Abdollahi et al., 2014; Fig. 7C): visual areas V4t and LO1, MST, MT, FST, and the putative human homolog of the posterior inferior temporal region (phPIT; Kolster et al., 2010) overlapped with the posterior part of this region. A large proportion of this region lay anterior to retinotopically organized cortex.

For shading, we evaluated whether the right inferior temporal cluster was specific for the $3 \mathrm{D}$ level or overlapped with the maps obtained for the lower-order processes. At a lower threshold, performance on 2D shape-from-shading correlated with volume loss posteriorly to the $3 \mathrm{D}$ shape-from-shading cluster and there was some overlap between the right occipitotemporal cluster obtained for 3D-shape-from-shading at our preset threshold (voxel-level $p<0.005$, cluster-level $p<0.05$ corrected) and 2D shape-from-shading (voxel-level $p<0.005$ without cluster-level threshold; Fig. $9 B$, yellow). We did not find overlap with elementary feature extraction for luminance.

Next, we evaluated whether the volumetric correlations obtained for 3D shape-from-shading and 3D shape-from-disparity were specific for PCA compared with the two other patient groups. There was limited overlap in the anterior portion of the inferior temporal cluster with the map obtained in typical $\mathrm{AD}$ patients for the correlation between brain volume and 3D shapefrom-shading if we lowered the threshold in the $\mathrm{AD}$ patients to uncorrected $p<0.01$ (Fig. 10, yellow). We also investigated whether performance on $3 \mathrm{D}$ shape-from-shading and $3 \mathrm{D}$ shapefrom-disparity in DLBD and AD patients correlated with gray matter volume in the regions defined independently in PCA for these cues. A significant correlation was found in $\mathrm{AD}$ between error-in-depth for 3D shape-from-shading and gray matter volume in the right inferior temporal region obtained in PCA for this cue $(r=-0.626, p=0.017)$.

We also evaluated in DLBD and typical AD patients whether any regions outside of the regions involved in 3D shape-from-

\footnotetext{
Figure 11. Task-related fMRI: $3 D$ shape-from-shading network. $A$, Visual stimuli of the fMRI experiment. Shading stimuli: 3D shaded stimuli and two control conditions: 2D shaded-blob and $2 D$ pixel-scrambled stimuli. $B, t$-map for the extraction of $3 D$ shape-from-shading [conjunction of contrast 1 (3D shape-from-shading minus $2 \mathrm{D}$ shaded-blobs) and contrast 2 (3D shapefrom-shading minus $2 \mathrm{D}$ pixel-scrambled)]. Random-effects analysis (fMRI) in 18 healthy controls, threshold: voxel-level uncorrected $p<0.001$, cluster-level FWE-corrected $p<0.05$. The $t$-map is projected onto a surface rendering of the brain (Van Essen, 2005). The black outlines reflect the regions found by Georgieva et al. (2008) in healthy young volunteers using the same contrast. C, Superposition between the fMRI activity cluster during passive viewing of 3D shape-from-shading minus control in healthy controls (red) and the cluster where gray matter volume correlates with $3 \mathrm{D}$ shape-from-shading scores in PCA (green). Overlap is indicated in yellow. $D, E$, Mean percentage signal change (relative to the fixation condition) for 3D shading stimuli, $2 \mathrm{D}$ pixel-scrambled stimuli, and 2D shaded-blobs in healthy controls and 5 PCA patients in left ITG (D) and right ITG (E). Error bars indicate SEM.
}

shading and 3D shape-from-disparity in PCA correlated with 3D shape-from-shading and 3D shape-from-disparity at FWE corrected cluster-level $p<0.05$. The only such region was found in $\mathrm{AD}$ in the anterior cingulate for $3 \mathrm{D}$ shape-from-disparity $(12,42$, $19, z=4.22$, extent 2491, corrected $p=0.003$ ).

Finally, we evaluated whether the effect of scanner site on brain volumetry overlapped with the region where we found a correlation between 3D shape-from-shading and 3D shapefrom-disparity and brain volume. This was restricted to a very minor overlap for 3D shape-from-shading $(0.005 \%$ of the inferior temporal cluster) and for 3D shape-from-disparity ( $0.42 \%$ of the inferior temporal cluster).

\section{fMRI activity levels in the 3D-processing network}

Next, we overlaid directly the volumetric analysis of 3D shapefrom-shading in PCA with the known fMRI activity pattern for $3 \mathrm{D}$ shape from shading versus $2 \mathrm{D}$ controls in healthy controls. According to online infrared eye tracking, participants kept stable gaze fixation on the central fixation cross. In healthy controls, 3D shape-from-shading activated the caudal ITG bilaterally (left ITG: $-45,-73,-8 ; z=6.86$; extent $5993 \times 3 \times 3 \mathrm{~mm}^{3}$ voxels; cluster-level $p<0.001$; right ITG: $45,-70,-11 ; z=5.28$; 346 voxels; Fig. $11 B$ ). The right ITG activation cluster overlapped with the region described previously by Georgieva et al. (2008) (marked by black outline in Fig. 11B) and with the region where volume loss correlated with performance of 3D shape-fromshading by PCA subjects (Fig. 11C, overlap marked in yellow). In the controls, another extensive activity cluster for 3D shapefrom-shading was present in the left anterior intraparietal sulcus (IPS) extending into the supramarginal gyrus $(-48,-34,43 ; z=$ 4.92; 447 voxels; $p<0.001$; Fig. $11 B$ ).

Finally, we looked for functional alterations in the $3 \mathrm{D}$ shapefrom-shading network due to PCA over and above what one would expect based on brain volume. We compared the average fMRI activity levels in each of the clusters that were activated in healthy subjects, controlling for gray matter volume as a covariate of no interest (Crawford et al., 2011). In 4 of 5 PCA patients, the effect size for contrast 1 (3D shape-from-shading minus 2D pixel-scrambled) in left posterior ITG was significantly smaller than in healthy controls $(p<=0.05)$. In right ITG, the effect size was reduced in 2 of 5 patients ( $p<=0.05$; Crawford et al., 2011). No significant effects were found in the IPS cluster. Overall, compared with baseline, the response in PCA patients compared with controls was reduced in both ITGs, in particular in the right ITG (Fig. $11 D, E$ ).

\section{Discussion}

In PCA patients, deficits on 3D shape-from-shading were associated with volume loss in right posterior inferior temporal cortex and deficits on 3D shape-from-disparity with volume loss slightly more anteriorly and in the right premotor cortex (Fig. 7). These findings obtained in PCA can be readily understood in the light of current neurophysiologically based models of 3D shape processing developed based on nonhuman primate research and fMRI in the intact human brain for these cues (Orban, 2011). They may also bear relevance for the far more common amnestic predominant forms of AD.

\section{Potential limitations}

PCA is characterized by a complex constellation of visuoperceptual and visuospatial disturbances (Benson et al., 1988; Hof et al., 1990; Graff-Radford et al., 1993; Levine et al., 1993; Giovagnoli et al., 2009). Nevertheless, we are confident that our psychophysical 
estimates reliably reflected the perceptual processes of interest rather than dysfunction in other domains: Subjects could only take part if they had passed a test session and proven that they were able to perform the $3 \mathrm{D}$ shape task reliably, leading to the exclusion of three patients. Furthermore, the response modality was flexibly adapted to the individual's capabilities based on the test session (key press, pointing, and vocal responses). Third, in 11 of 13 PCA patients, the 3D shape threshold was within normal limits for at least one cue (Table 2), most frequently the disparity cues (6/11 patients). The relative preservation of $3 \mathrm{D}$ shape from any cue provides evidence that the deficits observed were not caused by generic task demands. Fourth, the choice of global maximum by the PCA patients differed significantly from a random distribution or from what one would obtain in case of a random motor control error. For the lower-order tasks, further support is provided by the average staircase curves in the different populations. A reliable plateau level was reached, except for 2D-from-motion, in which the curve indicated a floor effect (Fig. 4). As a final argument, our main result, inferior temporal volume loss in relation to $3 \mathrm{D}$ shape processing (Fig. 7), is unlikely to be explained by manuomotor or visuomotor deficits (such as optic ataxia or gaze apraxia), which are typically localized to the dorsal stream.

Error-in-depth was abnormal only for monocular cues. For 3D shape-from-disparity, between-subjects variance in healthy controls was larger than for the other cues (Fig. 2). The carrier texture for 3D from disparity had the same resolution as that used for motion so that carrier texture cannot account for this difference. A possible explanation is the deterioration and increased variability in stereoacuity with normal aging (Bohr and Read, 2013). The analysis of the in-plane distribution of responses (Todd et al., 2001; Koenderink et al., 2002) clearly had added value in this respect and also revealed abnormal dispersion of responses in PCA patients for disparity.

\section{Neuroanatomical correlates of deficits in 3D shape perception}

Performance on 3D shape-from-shading correlated with right posterior inferior temporal volume loss (Fig. 7A). A separate fMRI experiment that we conducted in healthy older adults established the overlap between the fMRI focus activated by $3 \mathrm{D}$ shape-from-shading and the correlation between volume and 3D shape-from-shading performance in PCA patients (Fig. 11C). This cluster encompassed a caudal inferior temporal region that has been implicated previously in 3D shape-from-shading and shape-from-disparity by fMRI in the intact brain (Georgieva et al., 2008).

Comparison between this ITG region obtained in patients and the maximum probability map of retinotopic areas (Fig. 7C; Abdollahi et al., 2014) localizes the peak of this cluster to the rostral tip of the putative human homolog of the dorsal phPIT (phPITd; Kolster et al., 2010; Abdollahi et al., 2014) and neighboring retinotopic areas. In healthy human subjects, the local maximum of the $3 \mathrm{D}$ shape-from-shading activation is also located close to the rostral tip of the phPITd (Orban et al., 2014). Anteriorly, the volumetric cluster extended beyond retinotopic areas. In monkey fMRI, 3D shape-from-shading specifically activates V4 bilaterally and the dorsal portion of TEO (Boussaoud et al., 1991; Distler et al., 1993; Nelissen et al., 2009), now recognized as retinotopic PITd (Kolster et al., 2009, 2014). However, more widespread activation in FST and surrounding STS regions has also been reported using more naturalistic stimuli (Sereno et al., 2002).

In the right ITG region, the correlation with volume loss was not limited to 3D shape-from-shading (Fig. 7). There was also a correlation between 2D luminance processing and volume in part of this cluster. This was mainly due to overlap between the posterior part of the cluster for 3D shape-from-shading and that obtained for 2D luminance (Fig. 9B). Areas involved in 3D shapefrom-shading (principally area phPITd) may be close to areas involved in 2D orientation processing (such as the homolog of area V4; De Weerd et al., 1996), which may provide the input for the extraction of 3D shape (Fleming et al., 2011).

Slightly more anteriorly to the inferior temporal cluster obtained for 3D shape-from-shading, volume loss was associated with 3D shape-from-disparity. Performance on 3D shape-fromdisparity correlated with volume loss, not only in right posterior inferior temporal, but also in right premotor cortex. Numerous previous studies have indicated that the $3 \mathrm{D}$-processing network for binocular disparity is more extensive than the $3 \mathrm{D}$ networks for any of the monocular cues: in addition to ventral occipitotemporal areas (Janssen et al., 1999, 2000a, 2000b), it includes the IPS over its entire extent and even premotor cortex (Durand et al., 2007; Georgieva et al., 2009; Joly et al., 2009; Theys et al., 2012; Van Dromme et al., 2015). The premotor region where we find a correlation between volume loss and 3D shape-from-disparity fits well with these earlier observations (Georgieva et al., 2009) and likely encompasses the putative human homolog of F5 (Ferri et al., 2015). One of its subdivisions, area F5a, has been consistently found to be activated during 3D shape processing for disparity in fMRI in nonhuman primates (Joly et al., 2009; Theys et al., 2012), as well as in the intact human brain (Georgieva et al., 2009).

For two cues, motion and texture, 3D shape-processing deficits were present behaviorally, but without clear neuroanatomical substrate. These cues were among the most severely impaired. The areas implicated in 3D shape-from-motion, such as MT and FST (Vanduffel et al., 2002), were clearly affected in PCA patients (Fig. 5). The same holds true for the occipitotemporal regions involved in 3D shape-from-texture (Georgieva et al., 2009). Further work is needed to understand the lack of correlation for the motion and texture cues, which may reflect the organization (serial vs parallel) of the network extracting $3 \mathrm{D}$ shape from these cues, severity of atrophy, or the relatively small sample size combined with stringent thresholds applied to the whole-brain search volume.

\section{Relevance for other patient groups}

The atrophy pattern in extrastriate cortex was specific for PCA patients. Accordingly, the association between posterior inferior temporal volume loss and 3D shape-from-shading and shapefrom-disparity deficits was also relatively specific for PCA. The profound 3D shape-processing deficits in DLBD patients (Figs. 2, 3 ) were not associated with posterior inferior temporal volume loss. This indicates that structural integrity of right posterior inferior temporal cortex is not the sole factor determining success on these 3D shape-processing tasks. A previous volumetric MRI study in DLBD failed to reveal neocortical volume loss (Whitwell et al., 2007). In contrast, functional changes as measured with FDG-PET (Minoshima et al., 2001) or task-related fMRI (Taylor et al., 2012) frequently have been reported posteriorly. The visuoperceptual deficits in DLBD patients have been hypothetically attributed to loss of dopaminergic or cholinergic input (Whitwell et al., 2007), disconnection of the inferior longitudinal fascicle (Nedelska et al., 2015), or dopaminergic deficits in the retina (Mori et al., 2000) rather than structural volume loss.

In patients with the amnestic-predominant presentation of $\mathrm{AD}$, we found $3 \mathrm{D}$ shape perception deficits, although in a miti- 
gated form (Figs. 2, 3). Conceivably, 3D-processing deficits may go unnoticed in $\mathrm{AD}$ patients because they are overshadowed by the concomitant cognitive deficits or because gradual changes in visual perception may remain below the awareness level as long as they do not affect instrumental activities directly, as they do in PCA patients. Gray matter volume loss in AD extended into the anterior border of the right inferior temporal region implicated in 3D shape-from-shading in PCA. The distribution of NFTs in typical $\mathrm{AD}$ may partly extend into the anterior portion of the inferotemporal region implicated in 3D shape perception in PCA (Lewis et al., 1987; Braak et al., 1989). Gray matter volume in the right inferior temporal region identified in PCA also correlated with performance on this task in AD. Accordingly, the 3D shapefrom-shading deficit in $\mathrm{AD}$ may partly rely on a same substrate as that underlying 3D shape-processing deficits in PCA. However, other mechanisms may also contribute to the 3D shapeprocessing deficits in $\mathrm{AD}$, for example, at a postperceptual level. The correlation between anterior cingulate volume loss and 3D shape-from-disparity scores in $\mathrm{AD}$ could point in that direction given the role of this region in decision making (Walton et al., 2007).

\section{Conclusion}

According to contemporary models of the functional anatomy of $3 \mathrm{D}$ processing, 3D shape-from-shading principally relies on posterior inferior temporal cortex. In contrast, 3D shape-fromdisparity relies on widely distributed regions, not only in the ventral stream, but also in the dorsal stream extending into premotor cortex. The data that we obtained in patients with neurodegeneration targeting the ventral and dorsal processing stream are consistent with predictions based on the functional anatomical models derived from nonhuman primate research and fMRI of the intact brain. However, the 3D shape-processing deficits in DLBD remain unexplained at this stage and indicate that the structural integrity of right inferior temporal cortex cannot be the sole factor determining success on these $3 \mathrm{D}$ shape-processing tasks.

\section{References}

Abdollahi RO, Kolster H, Glasser MF, Robinson EC, Coalson TS, Dierker D, Jenkinson M, Van Essen DC, Orban GA (2014) Correspondences between retinotopic areas and myelin maps in human visual cortex. Neuroimage 99:509-524. CrossRef Medline

Ashburner J, Friston KJ (2005) Unified segmentation. Neuroimage 26:839851. CrossRef Medline

Benson DF, Davis RJ, Snyder BD (1988) Posterior cortical atrophy. Arch Neurol 45:789-793. CrossRef Medline

Berthier ML, Leiguarda R, Starkstein SE, Sevlever G, Taratuto AL (1991) Alzheimer's disease in a patient with posterior cortical atrophy. J Neurol Neurosurg Psychiatry 54:1110-1111. CrossRef Medline

Bohr I, Read JC (2013) Stereoacuity with frisby and revised fd2 stereo tests. PLoS One 8:e82999. CrossRef Medline

Boussaoud D, Desimone R, Ungerleider LG (1991) Visual topography of area teo in the macaque. J Comp Neurol 306:554-575. CrossRef Medline

Braak H, Braak E, Kalus P (1989) Alzheimer's disease: areal and laminar pathology in the occipital isocortex. Acta Neuropathol 77:494-506. CrossRef Medline

Caine D (2004) Posterior cortical atrophy: a review of the literature. Neurocase 10:382-385. CrossRef Medline

Calderon J, Perry RJ, Erzinclioglu SW, Berrios GE, Dening TR, Hodges JR (2001) Perception, attention, and working memory are disproportionately impaired in dementia with Lewy bodies compared with Alzheimer's disease. J Neurol Neurosurg Psychiatry 70:157-164. CrossRef Medline

Crawford JR, Garthwaite PH (2002) Investigation of the single case in neuropsychology: confidence limits on the abnormality of test scores and test score differences. Neuropsychologia 40:1196-1208. CrossRef Medline

Crawford JR, Garthwaite PH (2005) Testing for suspected impairments and dissociations in single-case studies in neuropsychology: Evaluation of alternatives using Monte Carlo simulations and revised tests for dissociations. Neuropsychology 19:318-331. CrossRef Medline

Crawford JR, Garthwaite PH, Ryan K (2011) Comparing a single case to a control sample: testing for neuropsychological deficits and dissociations in the presence of covariates. Cortex 47:1166-1178. CrossRef Medline

Crutch SJ, Lehmann M, Schott JM, Rabinovici GD, Rossor MN, Fox NC (2012) Posterior cortical atrophy. Lancet Neurol 11:170-178. CrossRef Medline

de Souza LC, Corlier F, Habert MO, Uspenskaya O, Maroy R, Lamari F, Chupin M, Lehéricy S, Colliot O, Hahn-Barma V, Samri D, Dubois B, Bottlaender M, Sarazin M (2011) Similar amyloid- $\beta$ burden in posterior cortical atrophy and Alzheimer's disease. Brain 134:2036-2043. CrossRef Medline

De Valois RL, Albrecht DG, Thorell LG (1982) Spatial frequency selectivity of cells in macaque visual cortex. Vision Res 22:545-559. CrossRef Medline

De Weerd P, Desimone R, Ungerleider LG (1996) Cue-dependent deficits in grating orientation discrimination after $\mathrm{v} 4$ lesions in macaques. Vis Neurosci 13:529-538. CrossRef Medline

Distler C, Boussaoud D, Desimone R, Ungerleider LG (1993) Cortical connections of inferior temporal area teo in macaque monkeys. J Comp Neurol 334:125-150. CrossRef Medline

Dubois B, Feldman HH, Jacova C, Hampel H, Molinuevo JL, Blennow K, DeKosky ST, Gauthier S, Selkoe D, Bateman R, Cappa S, Crutch S, Engelborghs S, Frisoni GB, Fox NC, Galasko D, Habert MO, Jicha GA, Nordberg A, Pasquier F, Rabinovici G, Robert P, Rowe C, Salloway S, Sarazin M, Epelbaum S, de Souza LC, Vellas B, Visser PJ, Schneider L, Stern Y, Scheltens P, Cummings JL (2014) Advancing research diagnostic criteria for Alzheimer's disease: the iwg-2 criteria. Lancet Neurol 13:614-629. CrossRef Medline

Dupont P, De Bruyn B, Vandenberghe R, Rosier AM, Michiels J, Marchal G, Mortelmans L, Orban GA (1997) The kinetic occipital region in human visual cortex. Cereb Cortex 7:283-292. CrossRef Medline

Durand JB, Nelissen K, Joly O, Wardak C, Todd JT, Norman JF, Janssen P, Vanduffel W, Orban GA (2007) Anterior regions of monkey parietal cortex process visual 3d shape. Neuron 55:493-505. CrossRef Medline

El-Shamayleh Y, Movshon JA (2011) Neuronal responses to texturedefined form in macaque visual area v2. J Neurosci 31:8543-8555. CrossRef Medline

Ferri S, Peeters R, Nelissen K, Vanduffel W, Rizzolatti G, Orban GA (2015) A human homologue of monkey f5c. Neuroimage 111:251-266. CrossRef Medline

Fleming RW, Torralba A, Adelson EH (2004) Specular reflections and the perception of shape. J Vis 4:798-820. CrossRef Medline

Fleming RW, Holtmann-Rice D, Bülthoff HH (2011) Estimation of 3d shape from image orientations. Proc Natl Acad Sci U S A 108:2043820443. CrossRef Medline

Georgieva SS, Todd JT, Peeters R, Orban GA (2008) The extraction of 3d shape from texture and shading in the human brain. Cereb Cortex 18: 2416-2438. CrossRef Medline

Georgieva S, Peeters R, Kolster H, Todd JT, Orban GA (2009) The processing of three-dimensional shape from disparity in the human brain. J Neurosci 29:727-742. Medline

Gillebert CR, Mantini D, Thijs V, Sunaert S, Dupont P, Vandenberghe R (2011) Lesion evidence for the critical role of the intraparietal sulcus in spatial attention. Brain 134:1694-1709. CrossRef Medline

Giovagnoli AR, Aresi A, Reati F, Riva A, Gobbo C, Bizzi A (2009) The neuropsychological and neuroradiological correlates of slowly progressive visual agnosia. Neurol Sci 30:123-131. CrossRef Medline

Graff-Radford NR, Bolling JP, Earnest F 4th, Shuster EA, Caselli RJ, Brazis PW (1993) Simultanagnosia as the initial sign of degenerative dementia. Mayo Clin Proc 68:955-964. CrossRef Medline

Hof PR, Bouras C (1991) Object recognition deficit in Alzheimer's disease: possible disconnection of the occipito-temporal component of the visual system. Neurosci Lett 122:53-56. CrossRef Medline

Hof PR, Bouras C, Constantinidis J, Morrison JH (1990) Selective disconnection of specific visual association pathways in cases of Alzheimer's disease presenting with balint's syndrome. J Neuropathol Exp Neurol 49:168-184. CrossRef Medline

Hof PR, Vogt BA, Bouras C, Morrison JH (1997) Atypical form of Alzheimer's disease with prominent posterior cortical atrophy: a review of lesion 
distribution and circuit disconnection in cortical visual pathways. Vision Res 37:3609-3625. CrossRef Medline

Janssen P, Vogels R, Orban GA (1999) Macaque inferior temporal neurons are selective for disparity-defined three-dimensional shapes. Proc Natl Acad Sci U S A 96:8217-8222. CrossRef Medline

Janssen P, Vogels R, Orban GA (2000a) Selectivity for 3d shape that reveals distinct areas within macaque inferior temporal cortex. Science 288: 2054-2056. CrossRef Medline

Janssen P, Vogels R, Orban GA (2000b) Three-dimensional shape coding in inferior temporal cortex. Neuron 27:385-397. CrossRef Medline

Joly O, Vanduffel W, Orban GA (2009) The monkey ventral premotor cortex processes $3 \mathrm{~d}$ shape from disparity. Neuroimage 47:262-272. CrossRef Medline

Kas A, de Souza LC, Samri D, Bartolomeo P, Lacomblez L, Kalafat M, Migliaccio R, Thiebaut de Schotten M, Cohen L, Dubois B, Habert MO, Sarazin M (2011) Neural correlates of cognitive impairment in posterior cortical atrophy. Brain 134:1464-1478. CrossRef Medline

Koenderink JJ, van Doorn AJ, Kappers AM, Todd JT (2002) Pappus in optical space. Percept Psychophys 64:380-391. CrossRef Medline

Kolster H, Mandeville JB, Arsenault JT, Ekstrom LB, Wald LL, Vanduffel W (2009) Visual field map clusters in macaque extrastriate visual cortex. J Neurosci 29:7031-7039. CrossRef Medline

Kolster H, Peeters R, Orban GA (2010) The retinotopic organization of the human middle temporal area $\mathrm{mt} / \mathrm{v} 5$ and its cortical neighbors. J Neurosci 30:9801-9820. CrossRef Medline

Kolster H, Janssens T, Orban GA, Vanduffel W (2014) The retinotopic organization of macaque occipitotemporal cortex anterior to v4 and caudoventral to the middle temporal (mt) cluster. J Neurosci 34:1016810191. CrossRef Medline

Kourtzi Z, Erb M, Grodd W, Bülthoff HH (2003) Representation of the perceived 3-d object shape in the human lateral occipital complex. Cereb Cortex 13:911-920. CrossRef Medline

Lehmann M, Barnes J, Ridgway GR, Wattam-Bell J, Warrington EK, Fox NC, Crutch SJ (2011a) Basic visual function and cortical thickness patterns in posterior cortical atrophy. Cereb Cortex 21:2122-2132. CrossRef Medline

Lehmann M, Crutch SJ, Ridgway GR, Ridha BH, Barnes J, Warrington EK, Rossor MN, Fox NC (2011b) Cortical thickness and voxel-based morphometry in posterior cortical atrophy and typical Alzheimer's disease. Neurobiol Aging 32:1466-1476. CrossRef Medline

Lehmann M, Madison CM, Ghosh PM, Seeley WW, Mormino E, Greicius MD, Gorno-Tempini ML, Kramer JH, Miller BL, Jagust WJ, Rabinovici GD (2013a) Intrinsic connectivity networks in healthy subjects explain clinical variability in Alzheimer's disease. Proc Natl Acad Sci U S A 110: 11606-11611. CrossRef Medline

Lehmann M, Ghosh PM, Madison C, Laforce R Jr, Corbetta-Rastelli C, Weiner MW, Greicius MD, Seeley WW, Gorno-Tempini ML, Rosen HJ, Miller BL, Jagust WJ, Rabinovici GD (2013b) Diverging patterns of amyloid deposition and hypometabolism in clinical variants of probable Alzheimer's disease. Brain 136:844-858. CrossRef Medline

Levine DN, Lee JM, Fisher CM (1993) The visual variant of Alzheimer's disease: a clinicopathologic case study. Neurology 43:305-313. CrossRef Medline

Lewis DA, Campbell MJ, Terry RD, Morrison JH (1987) Laminar and regional distributions of neurofibrillary tangles and neuritic plaques in Alzheimer's disease: a quantitative study of visual and auditory cortices. J Neurosci 7:1799-1808. Medline

McKeith IG, Dickson DW, Lowe J, Emre M, O’Brien JT, Feldman H, Cummings J, Duda JE, Lippa C, Perry EK, Aarsland D, Arai H, Ballard CG, Boeve B, Burn DJ, Costa D, Del Ser T, Dubois B, Galasko D, Gauthier S, Goetz CG, Gomez-Tortosa E, Halliday G, Hansen LA, Hardy J, Iwatsubo T, Kalaria RN, Kaufer D, Kenny RA, Korczyn A, Kosaka K, Lee VM, Lees A, Litvan I, Londos E, Lopez OL, Minoshima S, Mizuno Y, Molina JA, Mukaetova-Ladinska EB, Pasquier F, Perry RH, Schulz JB, Trojanowski JQ, Yamada M, Yamada M (2005) Diagnosis and management of dementia with lewy bodies: third report of the dlb consortium. Neurology 65:1863-1872. CrossRef Medline

McKhann GM, Knopman DS, Chertkow H, Hyman BT, Jack CR Jr, Kawas CH, Klunk WE, Koroshetz WJ, Manly JJ, Mayeux R, Mohs RC, Morris JC, Rossor MN, Scheltens P, Carrillo MC, Thies B, Weintraub S, Phelps CH (2011) The diagnosis of dementia due to Alzheimer's disease: Recommendations from the national institute on aging-Alzheimer's association workgroups on diagnostic guidelines for Alzheimer's disease. Alzheimers Dement 7:263-269. CrossRef Medline

Mendola JD, Dale AM, Fischl B, Liu AK, Tootell RB (1999) The representation of illusory and real contours in human cortical visual areas revealed by functional magnetic resonance imaging. J Neurosci 19:8560-8572. Medline

Michaelson A (1927) Studies in optics. Chicago: University of Chicago.

Migliaccio R, Agosta F, Scola E, Magnani G, Cappa SF, Pagani E, Canu E, Comi G, Falini A, Gorno-Tempini ML, Bartolomeo P, Filippi M (2012) Ventral and dorsal visual streams in posterior cortical atrophy: a dt mri study. Neurobiol Aging 33:2572-2584. CrossRef Medline

Minoshima S, Foster NL, Sima AA, Frey KA, Albin RL, Kuhl DE (2001) Alzheimer's disease versus dementia with lewy bodies: cerebral metabolic distinction with autopsy confirmation. Ann Neurol 50:358-365. CrossRef Medline

Moore C, Engel SA (2001) Neural response to perception of volume in the lateral occipital complex. Neuron 29:277-286. CrossRef Medline

Mori E, Shimomura T, Fujimori M, Hirono N, Imamura T, Hashimoto M, Tanimukai S, Kazui H, Hanihara T (2000) Visuoperceptual impairment in dementia with lewy bodies. Arch Neurol 57:489-493. CrossRef Medline

Mosimann UP, Mather G, Wesnes KA, O’Brien JT, Burn DJ, McKeith IG (2004) Visual perception in Parkinson disease dementia and dementia with Lewy bodies. Neurology 63:2091-2096. CrossRef Medline

Mysore SG, Vogels R, Raiguel SE, Orban GA (2006) Processing of kinetic boundaries in macaque v4. J Neurophysiol 95:1864-1880. CrossRef Medline

Mysore SG, Vogels R, Raiguel SE, Todd JT, Orban GA (2010) The selectivity of neurons in the macaque fundus of the superior temporal area for three-dimensional structure from motion. J Neurosci 30:15491-15508. CrossRef Medline

Nedelska Z, Ferman TJ, Boeve BF, Przybelski SA, Lesnick TG, Murray ME, Gunter JL, Senjem ML, Vemuri P, Smith GE, Geda YE, Graff-Radford J, Knopman DS, Petersen RC, Parisi JE, Dickson DW, Jack CR Jr, Kantarci K (2015) Pattern of brain atrophy rates in autopsy-confirmed dementia with lewy bodies. Neurobiol Aging 36:452-461. CrossRef Medline

Nelissen K, Joly O, Durand JB, Todd JT, Vanduffel W, Orban GA (2009) The extraction of depth structure from shading and texture in the macaque brain. PLoS One 4:e8306. CrossRef Medline

Norman JF, Todd JT (1995) The perception of 3-d structure from contradictory optical patterns. Percept Psychophys 57:826-834. CrossRef Medline

Norman JF, Todd JT, Orban GA (2004) Perception of three-dimensional shape from specular highlights, deformations of shading, and other types of visual information. Psychol Sci 15:565-570. CrossRef Medline

Orban GA (2011) The extraction of 3d shape in the visual system of human and nonhuman primates. Annu Rev Neurosci 34:361-388. CrossRef Medline

Orban GA, Zhu Q, Vanduffel W (2014) The transition in the ventral stream from feature to real-world entity representations. Front Psychol 5:695. Medline

Ossenkoppele R, Schonhaut DR, Baker SL, O’Neil JP, Janabi M, Ghosh PM, Santos M, Miller ZA, Bettcher BM, Gorno-Tempini ML, Miller BL, Jagust WJ, Rabinovici GD (2015) Tau, amyloid, and hypometabolism in a patient with posterior cortical atrophy. Ann Neurol 77:338-342. CrossRef Medline

Poline JB, Worsley KJ, Evans AC, Friston KJ (1997) Combining spatial extent and peak intensity to test for activations in functional imaging. Neuroimage 5:83-96. CrossRef Medline

Rogelet P, Delafosse A, Destee A (1996) Posterior cortical atrophy: unusual feature of Alzheimer's disease. Neurocase 2:495-501. CrossRef

Rosenbloom MH, Alkalay A, Agarwal N, Baker SL, O’Neil JP, Janabi M, Yen IV, Growdon M, Jang J, Madison C, Mormino EC, Rosen HJ, GornoTempini ML, Weiner MW, Miller BL, Jagust WJ, Rabinovici GD (2011) Distinct clinical and metabolic deficits in pca and ad are not related to amyloid distribution. Neurology 76:1789-1796. CrossRef Medline

Sáry G, Vogels R, Kovács G, Orban GA (1995) Responses of monkey inferior temporal neurons to luminance-, motion-, and texture-defined gratings. J Neurophysiol 73:1341-1354. Medline

Sereno ME, Trinath T, Augath M, Logothetis NK (2002) Three-dimensional shape representation in monkey cortex. Neuron 33:635-652. CrossRef Medline 
Sáry G, Vogels R, Orban GA (1994) Orientation discrimination of motiondefined gratings. Vision Res 34:1331-1334. CrossRef Medline

Tang-Wai DF, Graff-Radford NR, Boeve BF, Dickson DW, Parisi JE, Crook R, Caselli RJ, Knopman DS, Petersen RC (2004) Clinical, genetic, and neuropathologic characteristics of posterior cortical atrophy. Neurology 63: 1168-1174. CrossRef Medline

Taylor KI, Devereux BJ, Acres K, Randall B, Tyler LK (2012) Contrasting effects of feature-based statistics on the categorisation and basic-level identification of visual objects. Cognition 122:363-374. CrossRef Medline

Theys T, Pani P, van Loon J, Goffin J, Janssen P (2012) Selectivity for threedimensional shape and grasping-related activity in the macaque ventral premotor cortex. J Neurosci 32:12038-12050. CrossRef Medline

Todd JT (2004) The visual perception of 3d shape. Trends Cogn Sci 8:115121. CrossRef Medline

Todd JT, Oomes AH, Koenderink JJ, Kappers AM (2001) On the affine structure of perceptual space. Psychol Sci 12:191-196. CrossRef Medline

Tyler CW, Likova LT, Kontsevich LL, Wade AR (2006) The specificity of cortical region ko to depth structure. Neuroimage 30:228-238. CrossRef Medline

Van Dromme IC, Vanduffel W, Janssen P (2015) The relation between functional magnetic resonance imaging activations and single-cell selec- tivity in the macaque intraparietal sulcus. Neuroimage 113:86-100. CrossRef Medline

Van Essen DC (2005) A population-average, landmark- and surface-based (pals) atlas of human cerebral cortex. Neuroimage 28:635-662. CrossRef Medline

Vanduffel W, Fize D, Peuskens H, Denys K, Sunaert S, Todd JT, Orban GA (2002) Extracting 3D from motion: differences in human and monkey intraparietal cortex. Science 298:413-415. CrossRef Medline

von Gunten A, Bouras C, Kövari E, Giannakopoulos P, Hof PR (2006) Neural substrates of cognitive and behavioral deficits in atypical Alzheimer's disease. Brain Res Rev 51:176-211. CrossRef Medline

Walton ME, Croxson PL, Behrens TE, Kennerley SW, Rushworth MF (2007) Adaptive decision making and value in the anterior cingulate cortex. Neuroimage 36:T142-T154. CrossRef Medline

Watson AB, Pelli DG (1983) Quest: a bayesian adaptive psychometric method. Percept Psychophys 33:113-120. CrossRef Medline

Whitwell JL, Weigand SD, Shiung MM, Boeve BF, Ferman TJ, Smith GE, Knopman DS, Petersen RC, Benarroch EE, Josephs KA, Jack CR Jr (2007) Focal atrophy in dementia with Lewy bodies on MRI: a distinct pattern from Alzheimer's disease. Brain 130:708-719. CrossRef Medline 